\title{
Research Article \\ Existence Results for Quasilinear Elliptic Equations with Indefinite Weight
}

\author{
Mieko Tanaka \\ Department of Mathematics, Tokyo University of Science, Kagurazaka 1-3, Shinjuku-ku 162-8601, Japan \\ Correspondence should be addressed to Mieko Tanaka, tanaka@ma.kagu.tus.ac.jp
}

Received 19 January 2012; Accepted 7 March 2012

Academic Editor: Juan J. Nieto

Copyright (C 2012 Mieko Tanaka. This is an open access article distributed under the Creative Commons Attribution License, which permits unrestricted use, distribution, and reproduction in any medium, provided the original work is properly cited.

We provide the existence of a solution for quasilinear elliptic equation $-\operatorname{div}\left(a_{\infty}(x)|\nabla u|^{p-2} \nabla u+\right.$ $\tilde{a}(x,|\nabla u|) \nabla u)=\lambda m(x)|u|^{p-2} u+f(x, u)+h(x)$ in $\Omega$ under the Neumann boundary condition. Here, we consider the condition that $\widetilde{a}(x, t)=o\left(t^{p-2}\right)$ as $t \rightarrow+\infty$ and $f(x, u)=o\left(|u|^{p-1}\right)$ as $|u| \rightarrow \infty$. As a special case, our result implies that the following $p$-Laplace equation has at least one solution: $-\Delta_{p} u=\lambda m(x)|u|^{p-2} u+\mu|u|^{r-2} u+h(x)$ in $\Omega, \partial u / \partial v=0$ on $\partial \Omega$ for every $1<r<p<\infty, \lambda \in \mathbb{R}$, $\mu \neq 0$ and $m, h \in L^{\infty}(\Omega)$ with $\int_{\Omega} m d x \neq 0$. Moreover, in the nonresonant case, that is, $\lambda$ is not an eigenvalue of the $p$-Laplacian with weight $m$, we present the existence of a solution of the above $p$-Laplace equation for every $1<r<p<\infty, \mu \in \mathbb{R}$ and $m, h \in L^{\infty}(\Omega)$.

\section{Introduction}

In this paper, we consider the existence of a solution for the following quasilinear elliptic equation:

$$
\begin{gathered}
-\operatorname{div} A(x, \nabla u)=\lambda m(x)|u|^{p-2} u+f(x, u)+h(x) \quad \text { in } \Omega, \\
\frac{\partial u}{\partial v}=0 \quad \text { on } \partial \Omega,
\end{gathered}
$$$$
(P ; \lambda, m, h)
$$

where $\Omega \subset \mathbb{R}^{N}$ is a bounded domain with $C^{2}$ boundary $\partial \Omega, v$ denotes the outward unit normal vector on $\partial \Omega, \lambda \in \mathbb{R}, 1<p<\infty$ and $m, h \in L^{\infty}(\Omega)$. We assume that $f$ is a Carathéodory function on $\Omega \times \mathbb{R}$ satisfying

$$
\lim _{|t| \rightarrow \infty} \frac{f(x, t)}{|t|^{p-2} t}=0 \quad \text { uniformly in } x \in \Omega,
$$


and that $f(x, t)$ is bounded on a bounded set (admitting $f \equiv 0$ in the nonresonant case). Here, $A: \bar{\Omega} \times \mathbb{R}^{N} \rightarrow \mathbb{R}^{N}$ is a map which is strictly monotone in the second variable and satisfies certain regularity conditions (see the following assumption $(A)$ ). The equation $(P ; \lambda, m, h)$ contains the corresponding $p$-Laplacian problem as a special case. Although the operator $A$ is nonhomogeneous in the second variable in general, we assume that $A(x, y)$ is asymptotically $(p-1)$-homogeneous at infinity in the following sense $(A H)$.

Throughout this paper, we assume that the map $A$ satisfies the following assumptions $(A H)$ and $(A)$ :

$(A H)$ there exist a positive function $a_{\infty} \in C^{1}(\bar{\Omega}, \mathbb{R})$ and a continuous function $\tilde{a}(x, t)$ on $\bar{\Omega} \times \mathbb{R}$ such that

$$
\begin{gathered}
A(x, y)=a_{\infty}(x)|y|^{p-2} y+\tilde{a}(x,|y|) y \quad \text { for every } x \in \Omega, y \in \mathbb{R}^{N}, \\
\lim _{t \rightarrow+\infty} \frac{\tilde{a}(x, t)}{t^{p-2}}=0 \quad \text { uniformly in } x \in \bar{\Omega} .
\end{gathered}
$$

(A) $A(x, y)=a(x,|y|) y$, where $a(x, t)>0$ for all $(x, t) \in \bar{\Omega} \times(0,+\infty)$ and

(i) $A \in C^{0}\left(\bar{\Omega} \times \mathbb{R}^{N}, \mathbb{R}^{N}\right) \cap C^{1}\left(\bar{\Omega} \times\left(\mathbb{R}^{N} \backslash\{0\}\right), \mathbb{R}^{N}\right)$;

(ii) there exists $C_{1}>0$ such that

$$
\left|D_{y} A(x, y)\right| \leq C_{1}|y|^{p-2} \text { for every } x \in \bar{\Omega}, y \in \mathbb{R}^{N} \backslash\{0\} ;
$$

(iii) there exists $C_{0}>0$ such that

$$
D_{y} A(x, y) \xi \cdot \xi \geq C_{0}|y|^{p-2}|\xi|^{2} \quad \text { for every } x \in \bar{\Omega}, y \in \mathbb{R}^{N} \backslash\{0\}, \xi \in \mathbb{R}^{N}
$$

(iv) there exists $C_{2}>0$ such that

$$
\left|D_{x} A(x, y)\right| \leq C_{2}\left(1+|y|^{p-1}\right) \quad \text { for every } x \in \bar{\Omega}, y \in \mathbb{R}^{N} \backslash\{0\} .
$$

A similar hypothesis to $(A)$ is considered in the study of quasilinear elliptic problems (cf. [1, Example 2.2], [2-6]). It is easily seen that many examples as in the above references satisfy the condition $(A H)$. Also, the following example satisfies our hypotheses:

$$
\operatorname{div}\left(\left(|\nabla u|^{p-2}+|\nabla u|^{q-2}\right)\left(1+|\nabla u|^{q}\right)^{(p-q) / q} \nabla u\right) \quad \text { for } 1<p \leq q<\infty
$$

In particular, for $A(x, y)=|y|^{p-2} y$, that is, $\operatorname{div} A(x, \nabla u)$ stands for the usual $p$-Laplacian $\Delta_{p} u$, we can take $C_{0}=C_{1}=p-1$ in $(A)$. Conversely, in the case where $C_{0}=C_{1}=p-1$ holds in $(A)$, by the inequalities in Remark 1.4 (ii) and (iii), we see $a(x, t)=|t|^{p-2}$ whence $A(x, y)=|y|^{p-2} y$.

Concerning the weight $m$, throughout this paper, we assume that

$$
|\{m>0\}|:=|\{x \in \Omega ; m(x)>0\}|>0
$$

holds, where $|X|$ denotes the Lebesgue measure of a measurable set $X$. 
Because $A(x, y)$ is asymptotically ( $p-1)$-homogeneous at infinity, the solvability of our equation is related to the following homogeneous equation (see Theorem 1.1):

$$
\begin{gathered}
-\operatorname{div}\left(a_{\infty}(x)|\nabla u|^{p-2} \nabla u\right)=\lambda m(x)|u|^{p-2} u \quad \text { in } \Omega, \\
\frac{\partial u}{\partial v}=0 \quad \text { on } \partial \Omega,
\end{gathered}
$$

where $a_{\infty}$ is the positive function as in $(A H)$. We say that $\lambda \in \mathbb{R}$ is an eigenvalue of $(E V ; m)$ if the equation $(E V ; m)$ has a nontrivial solution.

There are few existence results of a solution to our equation (and also the $p$-Laplace equation). For example, if $\lambda<0$ and $m \equiv 1$ hold, then the standard argument guarantees the existence of a solution. For the $p$-Laplacian as a special case of our problem, it is shown in [7] that the equation

$$
-\Delta_{p} u=\lambda m|u|^{p-2} u+h \quad \text { in } \Omega \quad \frac{\partial u}{\partial \nu}=0 \quad \text { on } \partial \Omega
$$

has a unique positive solution provided $0<\lambda<\lambda^{*}(m), \int_{\Omega} m d x<0$ and $0 \not \equiv h \in L^{\infty}(\Omega)_{+}$, where $\lambda^{*}(m)$ is the principal eigenvalue defined in Section 2.1 with $a_{\infty} \equiv 1$. In [8], although the resonant case where $\lambda=\lambda_{1}(m)$ or $\lambda=\lambda_{2}(m)$ is considered under the assumptions to $f(x, u)=f(u)$, its result does not cover the case of $f(u)=|u|^{r-2} u$ with $1<r<p$, where $\lambda_{i}(m)$ $(i=1,2)$ is $i$ th eigenvalue of the $p$-Laplacian with weight $m$. For the Laplace problem under the Neumann boundary condition, we can refer to $[9,10]$. Under the Dirichlet boundary condition, the existence results for the Laplace problem are well known when $m \equiv 1$ and $\lambda$ is not an eigenvalue of the Laplacian (cf. [11]). Moreover, under the Dirichlet (or blow-up) boundary condition, many authors study various equations involving the $p$-Laplace (Laplace) operator with (indefinite) weight. For example, we refer to [12] for boundary blow-up problems with Laplacian, [13] for periodic reaction-diffusion problems and [14, 15] for singular quasilinear elliptic problems.

Recently, the present author shows the existence of a solution for our problem in the case where $\lambda$ is between the principal eigenvalue and the second eigenvalue in [6] (for $f \equiv 0$ ). In addition, a similar situation is treated in [5]. However, existence results are not seen in the case when $\lambda$ is greater than the second eigenvalue for our problem. Therefore, the first purpose of this paper is to present an existence result of a solution in the nonresonant case where $\lambda$ is not an eigenvalue of $(E V ; m)$. Then, it studied the existence of at least one solution in the resonant case under assumptions that cover the case $f(u)=\mu|u|^{r-2} u$ with $1<r<p$ and $\mu \neq 0$.

For the proof of our result, it is necessary to study the weighted eigenvalue problem $(E V ; m)$. Thus, in Section 2 , we introduce two sequences $\left\{\lambda_{n}(m)\right\}_{n}$ and $\left\{\mu_{n}(m)\right\}_{n}$ of an eigenvalue of $(E V ; m)$ defined by Ljusternik-Schnirelman theory or Drábek-Robinson's method (cf. [16]), respectively. Then, we show several properties of above eigenvalues. In Section 3, we give the proof in the nonresonant case by using $\left\{\mu_{n}(m)\right\}_{n}$. In Sections 4 and 5 , we handle the resonant case. 


\subsection{Statements of Our Existence Results}

First, we state the existence result of a solution in the nonresonant case.

Theorem 1.1. Assume that $\lambda \in \mathbb{R}$ is not an eigenvalue of $(E V ; m)$. Then $(P ; \lambda, m, h)$ has at least one solution.

To state our existence result in the resonant case, we introduce some conditions. Set

$$
F(x, u):=\int_{0}^{u} f(x, s) d s, \quad \tilde{G}(x, y):=\int_{0}^{|y|} \tilde{a}(x, t) t d t
$$

where $\tilde{a}$ is the function as in $(A H)$.

$(H+)$ there exist $0 \leq q \leq p-1$ and $H_{0}>0$ such that

$$
\begin{aligned}
& \lim _{|y| \rightarrow \infty} \frac{p \tilde{G}(x, y)-\tilde{a}(x,|y|)|y|^{2}}{|y|^{1+q}}=+\infty \quad \text { uniformly in a.e. } x \in \Omega, \\
& f(x, t) t-p F(x, t) \geq-H_{0}\left(1+|t|^{1+q}\right) \quad \text { for a.e. } x \in \Omega, \text { every } t \in \mathbb{R} ;
\end{aligned}
$$

$(H-)$ there exist $0 \leq q \leq p-1$ and $H_{0}>0$ such that

$$
\begin{aligned}
& \lim _{|y| \rightarrow \infty} \frac{p \widetilde{G}(x, y)-\tilde{a}(x,|y|)|y|^{2}}{|y|^{1+q}}=-\infty \quad \text { uniformly in a.e. } x \in \Omega, \\
& f(x, t) t-p F(x, t) \leq H_{0}\left(|t|^{1+q}+1\right) \quad \text { for a.e. } x \in \Omega, \text { every } t \in \mathbb{R} ;
\end{aligned}
$$

$(H F+)$ there exist $0 \leq q \leq p-1$ and $H_{0}>0$ such that

$$
\begin{gathered}
p \tilde{G}(x, y)-\tilde{a}(x,|y|)|y|^{2} \geq-H_{0}\left(1+|y|^{1+q}\right) \quad \text { for every } x \in \Omega, y \in \mathbb{R}^{N}, \\
\lim _{|t| \rightarrow \infty} \frac{f(x, t) t-p F(x, t)}{|t|^{1+q}}=+\infty \quad \text { uniformly in a.e. } x \in \Omega
\end{gathered}
$$

$(H F-)$ there exist $0 \leq q \leq p-1$ and $H_{0}>0$ such that

$$
\begin{gathered}
p \tilde{G}(x, y)-\tilde{a}(x,|y|)|y|^{2} \leq H_{0}\left(1+|y|^{1+q}\right) \quad \text { for every } x \in \Omega, y \in \mathbb{R}^{N}, \\
\lim _{|t| \rightarrow \infty} \frac{f(x, t) t-p F(x, t)}{|t|^{1+q}}=-\infty \text { uniformly in a.e. } x \in \Omega .
\end{gathered}
$$


Theorem 1.2. Assume one of the following conditions:

(i) $\lambda=0$ and $(\mathrm{HF}+$ ) or (HF-) hold;

(ii) $\lambda \neq 0, \int_{\Omega} m d x \neq 0$ and one of $(H+),(H-),(H F+)$ and $(H F-)$ hold;

(iii) $\lambda \neq 0, \int_{\Omega} m d x=0$ and $(H+)$ or $(H F+)$ hold;

Then, $(P ; \lambda, m, h)$ has at least one solution.

In the special case where $\tilde{a}(x, t) \equiv 0$ and $f(x, u)=\mu|u|^{r-2} u$ for $1<r<p$, we easily see that $(H F+)$ or $(H F-)$ holds with $0 \leq q<r-1$ provided $\mu<0$ or $\mu>0$, respectively. Therefore, the following result is proved according to Theorem 1.2.

Corollary 1.3. Let $1<r<p<\infty, \mu \neq 0$ and $\int_{\Omega} m d x \neq 0$. Then, the following equation has at least one solution:

$$
\begin{gathered}
-\operatorname{div}\left(a_{\infty}(x)|\nabla u|^{p-2} \nabla u\right)=\lambda m(x)|u|^{p-2} u+\mu|u|^{r-2} u+h(x) \quad \text { in } \Omega, \\
\frac{\partial u}{\partial v}=0 \quad \text { on } \partial \Omega .
\end{gathered}
$$

\subsection{Properties of the Map $A$}

In what follows, the norm on $W^{1, p}(\Omega)$ is given by $\|u\|^{p}:=\|\nabla u\|_{p}^{p}+\|u\|_{p}^{p}$, where $\|u\|_{q}$ denotes the norm of $L^{q}(\Omega)$ for $u \in L^{q}(\Omega)(1 \leq q \leq \infty)$. Setting $G(x, y):=\int_{0}^{|y|} a(x, t) t d t$, then we can easily see that

$$
\nabla_{y} G(x, y)=A(x, y), \quad G(x, 0)=0
$$

for every $x \in \bar{\Omega}$.

Remark 1.4. It is easily seen that the following assertions hold under condition $(A)$ :

(i) for all $x \in \bar{\Omega}, A(x, y)$ is maximal monotone and strictly monotone in $y$;

(ii) $|A(x, y)| \leq\left(C_{1} /(p-1)\right)|y|^{p-1}$ for every $(x, y) \in \bar{\Omega} \times \mathbb{R}^{N}$;

(iii) $A(x, y) y \geq\left(C_{0} /(p-1)\right)|y|^{p}$ for every $(x, y) \in \bar{\Omega} \times \mathbb{R}^{N}$;

(iv) $G(x, y)$ is convex in $y$ for all $x$ and satisfies the following inequalities:

$$
A(x, y) y \geq G(x, y) \geq \frac{C_{0}}{p(p-1)}|y|^{p}, \quad G(x, y) \leq \frac{C_{1}}{p(p-1)}|y|^{p}
$$

for every $(x, y) \in \bar{\Omega} \times \mathbb{R}^{N}$, where $C_{0}$ and $C_{1}$ are the positive constants in $(A)$. 
The following result is proved in [3]. It plays an important role for our poof.

Proposition 1.5 (see [3, Proposition 1]). Let $A: W^{1, p}(\Omega) \rightarrow W^{1, p}(\Omega)^{*}$ be the map defined by

$$
\langle A(u), v\rangle=\int_{\Omega} A(x, \nabla u) \nabla v d x
$$

for $u, v \in W^{1, p}(\Omega)$. Then, $A$ has the $(S)_{+}$property, that is, any sequence $\left\{u_{n}\right\}$ weakly convergent to $u$ with $\lim \sup _{n \rightarrow \infty}\left\langle A\left(u_{n}\right), u_{n}-u\right\rangle \leq 0$ strongly converges to $u$.

\section{The Weighted Eigenvalue Problems}

\subsection{Preliminaries}

The following lemmas can be easily shown by way of contradiction because $\int_{\Omega} a_{\infty}|\nabla u|^{p} d x$ is equivalent to $\|\nabla u\|_{p}^{p}$ (note that $a_{\infty}$ is positive). Here, we omit the proofs (refer to [7]).

Lemma 2.1. Assume $\int_{\Omega} m d x<0$. Then, there exists a constant $c>0$ such that $\int_{\Omega} a_{\infty}|\nabla u|^{p} d x \geq$ $c\|u\|_{p}^{p}$ for every $u \in W^{1, p}(\Omega)$ with $\int_{\Omega} m|u|^{p} d x>0$.

Lemma 2.2. Assume that $\int_{\Omega} m d x \neq 0$ and $\xi>0$. Then, there exists a constant $b(m, \xi)>0$ such that

$$
\int_{\Omega} a_{\infty}|\nabla u|^{p} d x-\xi \int_{\Omega} m|u|^{p} d x \geq b(m, \xi) \int_{\Omega}|u|^{p} d x
$$

for every $u \in B(m):=\left\{u \in W^{1, p}(\Omega) ; \int_{\Omega} m|u|^{p} d x \leq 0\right\}$.

Lemma 2.3. Assume that $m \geq 0$ in $\Omega$. Then, for every $\xi>0$ there existed $d(m, \xi)>0$ such that

$$
\int_{\Omega} a_{\infty}|\nabla u|^{p} d x-\xi \int_{\Omega} m|u|^{p} d x \geq d(m, \xi) \int_{\Omega}|u|^{p} d x
$$

for every $u \in W^{1, p}(\Omega)$.

First, we recall the following principle eigenvalue $\lambda^{*}(m)$ :

$$
\lambda^{*}(m):=\inf \left\{\int_{\Omega} a_{\infty}|\nabla u|^{p} d x ; u \in W^{1, p}(\Omega), \int_{\Omega} m|u|^{p} d x=1\right\} .
$$

Because of $\infty>\sup _{x \in \Omega} a_{\infty}(x) \geq \inf _{x \in \Omega} a_{\infty}(x)>0$, we have the following result as the same argument as in the case of the $p$-Laplacian.

Proposition 2.4 (see [7, Proposition 2.2]). The following assertions hold:

(i) If $\int_{\Omega} m d x \geq 0$ holds, then $\lambda^{*}(m)=0$;

(ii) If $\int_{\Omega} m d x<0$ holds, then $\lambda^{*}(m)>0$ is a simple eigenvalue and it admits a positive eigenfunction. In addition, the open interval $\left(0, \lambda^{*}(m)\right)$ contains no eigenvalues of $(E V ; m)$. 
Lemma 2.5. Assume $\int_{\Omega} m d x<0$. Then, one has $\lambda^{*}(m+\varepsilon)<\lambda^{*}(m)<\lambda^{*}\left(m-\varepsilon^{\prime}\right)$ for every $\varepsilon>0$ and $\varepsilon^{\prime}>0$ with $\left|\left\{m-\varepsilon^{\prime}>0\right\}\right|>0$.

Proof. We choose a minimizer $u$ for $\lambda^{*}(m)$ because Proposition 2.4 guarantees the existence of it. Then, for every $\varepsilon>0$, we have

$$
\lambda^{*}(m+\varepsilon) \leq \frac{\int_{\Omega} a_{\infty}|\nabla u|^{p} d x}{\int_{\Omega}(m+\varepsilon)|u|^{p} d x}<\frac{\int_{\Omega} a_{\infty}|\nabla u|^{p} d x}{\int_{\Omega} m|u|^{p} d x}=\int_{\Omega} a_{\infty}|\nabla u|^{p} d x=\lambda^{*}(m)
$$

by the definition of $\lambda^{*}(m+\varepsilon)$. By applying the same argument to a minimizer for $\lambda^{*}(m-\varepsilon)$, we obtain $\lambda^{*}(m)<\lambda^{*}\left(m-\varepsilon^{\prime}\right)$ for $\varepsilon^{\prime}>0$ with $\left|\left\{m-\varepsilon^{\prime}>0\right\}\right|>0$.

\subsection{Other Eigenvalues}

Here, we introduce two unbounded sequences $\left\{\lambda_{n}(m)\right\}_{n}$ and $\left\{\mu_{n}(m)\right\}_{n}$ as follows:

$$
\begin{aligned}
J(u) & :=\int_{\Omega} a_{\infty}|\nabla u|^{p} d x \text { for } u \in W^{1, p}(\Omega), \quad \tilde{J}:=\left.J\right|_{S(m)}, \\
S(m) & :=\left\{u \in W^{1, p}(\Omega) ; \int_{\Omega} m|u|^{p} d x=1\right\}, \\
\mathcal{S}_{n}(m) & :=\{X \subset S(m) ; \text { compact, symmetric and } \gamma(X) \geq n\}, \\
\mathcal{F}_{n}(m) & :=\left\{g \in C\left(S^{n-1}, S(m)\right) ; g \text { is odd }\right\}, \\
\lambda_{n}(m) & :=\inf _{X \in \mathcal{S}_{n}(m)} \max _{u \in X} \tilde{J}(u), \\
\mu_{n}(m) & :=\inf _{g \in \mathcal{F}_{n}(m)} \max _{z \in S^{n-1}} \tilde{J}(g(z))
\end{aligned}
$$

where $\gamma(X)$ denotes the Krasnoselskii genus of $X$ (see [17, Definition 5.1] for the definition) and $S^{n-1}$ denotes the usual unit sphere in $\mathbb{R}^{n}$. We see that $\lambda_{n}(m)$ is defined by LjusternikSchnirelman theory and it is known that the definition of $\mu_{n}(m)$ is introduced by Drábek and Robinson ([16]) under the $p$-Laplace Dirichlet problem with $m \equiv 1$.

Remark 2.6. The following assertions can be shown easily:

(i) $\lambda_{1}(m)=\mu_{1}(m)=\lambda^{*}(m)$;

(ii) $\mathcal{S}_{n}(m) \neq \emptyset$ and $\mathcal{F}_{n}(m) \neq \emptyset$ for every $n \in \mathbb{N}$;

(iii) $g\left(S^{n-1}\right) \subset \mathcal{S}_{n}(m)$ for every $g \in \mathcal{F}_{n}(m)$;

(iv) $\mu_{n}(m) \geq \lambda_{n}(m)$ for every $n \in \mathbb{N}$;

(v) $\lambda_{n+1}(m) \geq \lambda_{n}(m)$ and $\mu_{n+1}(m) \geq \mu_{n}(m)$ for every $n \in \mathbb{N}$,

see [18] for the proof of (ii). 
Define a $C^{1}$ functional $\Phi_{m}$ on $W^{1, p}(\Omega)$ by $\Phi_{m}(u):=\int_{\Omega} m|u|^{p} d x$ for $u \in W^{1, p}(\Omega)$. Because $1 \in \mathbb{R}$ is a regular value of $\Phi_{m}$, it is well known that the norm of the derivative at $u \in S(m)$ of the restriction of $J$ to $S(m)$ is defined as follows:

$$
\begin{aligned}
\left\|\widetilde{J}^{\prime}(u)\right\|_{*} & :=\min \left\{\left\|J^{\prime}(u)-t \Phi_{m}^{\prime}(u)\right\|_{W^{1, p}(\Omega)^{*}} ; t \in \mathbb{R}\right\} \\
& =\sup \left\{\left\langle J^{\prime}(u), v\right\rangle ; v \in T_{u}(S(m)),\|v\|=1\right\},
\end{aligned}
$$

where $T_{u}(S(m))$ denotes the tangent space of $S(m)$ at $u$, that is, $T_{u}(S(m))=\left\{v \in W^{1, p}(\Omega)\right.$; $\left.\int_{\Omega} m|u|^{p-2} u v d x=0\right\}$. Here, we recall the definition of the Palais-Smale condition for $\tilde{J}$.

Definition 2.7. $\tilde{J}$ is said to satisfy the bounded Palais-Smale condition if any bounded sequence $u_{n} \in S(m)$ such that $\left\|\widetilde{J}^{\prime}\left(u_{n}\right)\right\|_{*} \rightarrow 0$ has a convergent subsequence. Moreover, we say that $\tilde{J}$ satisfies the Palais-Smale condition at level $c \in \mathbb{R}$ if any sequence $u_{n} \in S(m)$ such that $\tilde{J}\left(u_{n}\right) \rightarrow c$ and $\left\|\widetilde{J}^{\prime}\left(u_{n}\right)\right\|_{*} \rightarrow 0$ as $n \rightarrow \infty$ has a convergent subsequence. In addition, we say that $\tilde{J}$ satisfies the Palais-Smale condition if $\tilde{J}$ satisfies the Palais-Smale condition for every $c \in \mathbb{R}$.

The following result can be proved by the same argument as in [19, Proposition 3.3] (which treats the case of the $p$-Laplacian, i.e., $a_{\infty} \equiv 1$ ) because of $\infty>\sup _{x \in \Omega} a_{\infty}(x) \geq$ $\inf _{x \in \Omega} a_{\infty}(x)>0$. Here, we omit the proof.

Lemma 2.8. The following assertions hold:

(i) J satisfies the bounded Palais-Smale condition;

(ii) $\tilde{J}$ satisfies the Palais-Smale condition provided $\int_{\Omega} m d x \neq 0$.

Proposition 2.9. $\lambda_{n}(m)$ and $\mu_{n}(m)$ are eigenvalues of $(E V ; m)$ such that

$$
\lim _{n \rightarrow \infty} \lambda_{n}(m)=\lim _{n \rightarrow \infty} \mu_{n}(m)=+\infty
$$

Proof. In the case of $\int_{\Omega} m d x \neq 0$, since $\widetilde{J}$ satisfies the Palais-Smale condition, we can apply the first deformation lemma on $C^{1}$ manifold (refer to [20]). Thus, by the standard argument, we can prove that $\lambda_{n}(m)$ and $\mu_{n}(m)$ are critical values of $\tilde{J}$. This means that $\lambda_{n}(m)$ and $\mu_{n}(m)$ are eigenvalues of $(E V ; m)$ by the Lagrange multiplier rule. In addition, we can easily show $\lim _{n \rightarrow \infty} \lambda_{n}(m)=+\infty$ by the standard argument via the first deformation lemma on $C^{1}$ manifold (refer to [21, Proposition 3.14.7], [22] or [17] in the case of a Banach space). Hence, $\lim _{n \rightarrow \infty} \mu_{n}(m)=+\infty$ holds because of $\mu_{n}(m) \geq \lambda_{n}(m)$ for every $n \in \mathbb{N}$.

In the case of $\int_{\Omega} m d x=0$, by the same argument as in [18], our conclusion can be proved. For readers' convenience, we give a sketch of the proof. For $\varepsilon>0$, we define $J_{\varepsilon}(u):=$ $J(u)+\varepsilon\|u\|_{p}^{p}$ and $\tilde{J}_{\varepsilon}:=\left.J_{\varepsilon}\right|_{S(m)}$. Moreover, we set minimax values $\lambda_{n}^{\varepsilon}(m)$ and $\mu_{n}^{\varepsilon}(m)$ of $\widetilde{J}_{\varepsilon}$ by

$$
\lambda_{n}^{\varepsilon}(m):=\inf _{X \in \mathcal{S}_{n}(m)} \max _{u \in X} \tilde{J}_{\mathcal{\varepsilon}}(u), \quad \mu_{n}^{\varepsilon}(m):=\inf _{g \in \mathscr{F}_{n}(m)} \max _{z \in S^{n-1}} \tilde{J}_{\mathcal{\varepsilon}}(g(z)) .
$$

Because any Palais-Smale sequence of $\widetilde{J}_{\varepsilon}$ is bounded, it is easily shown that $\widetilde{J}_{\varepsilon}$ satisfies the Palais-Smale condition (refer to [19, Proposition 3.3]) Hence, it can be proved that $\lambda_{n}^{\varepsilon}(m)$ 
and $\mu_{n}^{\varepsilon}(m)$ are critical values of $\tilde{J}_{\varepsilon}$. Furthermore, it follows from the argument as in [18, Lemma 3.5] that $\lambda_{n}^{\varepsilon}(m) \rightarrow \lambda_{n}(m)$ and $\mu_{n}^{\varepsilon}(m) \rightarrow \mu_{n}(m)$ as $\varepsilon \rightarrow 0+$. Therefore, by noting that $J_{\varepsilon}$ is $p$-homogeneous, we can obtain a solution $u_{\varepsilon}$ with $\left\|u_{\varepsilon}\right\|=1$ for $-\operatorname{div}\left(a_{\infty}|\nabla u|^{p-2} \nabla u\right)=$ $c_{\varepsilon} m|u|^{p-2} u$ in $\Omega, \partial u / \partial v=0$ on $\partial \Omega$, where $c_{\varepsilon}=\lambda_{n}^{\varepsilon}(m)$ or $\mu_{n}^{\varepsilon}(m)$. Because of $\left\|u_{\varepsilon}\right\|=1$, it follows from the standard argument that $u_{\varepsilon}$ has a subsequence strongly convergent to a solution $u$ for

$$
-\operatorname{div}\left(a_{\infty}|\nabla u|^{p-2} \nabla u\right)=c m|u|^{p-2} u \quad \text { in } \Omega, \quad \frac{\partial u}{\partial v}=0 \quad \text { on } \partial \Omega,
$$

where $c=\lim _{\varepsilon \rightarrow 0+} \mathcal{C}_{\varepsilon}$. Thus, $\lambda_{n}(m)$ and $\mu_{n}(m)$ are eigenvalues of $(E V ; m)$. To prove $\lim _{n \rightarrow \infty} \lambda_{n}(m)=+\infty$, by considering a function $m_{\delta}(x):=\max \{m(x), \delta\}$ for $\delta>0$, we have $\lambda_{n}\left(m_{\delta}\right) \leq \lambda_{n}(m)$ (refer to Proposition 2.10). Because we can apply our fist assertion to $m_{\delta}$ (note $\int_{\Omega} m_{\delta} d x>0$ ), we obtain $\lim _{n \rightarrow \infty} \mu_{n}(m) \geq \lim _{n \rightarrow \infty} \lambda_{n}(m) \geq \lim _{n \rightarrow \infty} \lambda_{n}\left(m_{\delta}\right)=+\infty$.

Proposition 2.10. Let $1<r<\infty$ if $N \leq p$ and $p^{*} /\left(p^{*}-p\right) \leq r<\infty$ if $N>p$. Then, the following assertions hold:

(i) if $m^{\prime} \geq m$ in $\Omega$, then $\mu_{k}\left(m^{\prime}\right) \leq \mu_{k}(m)$;

(ii) if $\lim _{n \rightarrow \infty} m_{n}=m$ in $L^{r}(\Omega)$, then $\lim \sup _{n \rightarrow \infty} \mu_{k}\left(m_{n}\right) \leq \mu_{k}(m)$;

(iii) if $\int_{\Omega} m d x \neq 0$ and $\lim _{n \rightarrow \infty} m_{n}=m$ in $L^{r}(\Omega)$, then $\lim _{n \rightarrow \infty} \mu_{k}\left(m_{n}\right)=\mu_{k}(m)$.

Moreover, the same conclusion holds for $\lambda_{k}(m)$.

Proof. We only treat $\mu_{k}(m)$ because we can give the proof for $\lambda_{k}(m)$ similarly.

(i) Let $m^{\prime} \geq m$ in $\Omega$. Fix an arbitrary $\varepsilon>0$. Then, by the definition of $\mu_{k}(m)$, there exists a $g \in \mathcal{F}_{k}(m)$ such that $\max _{z \in S^{k-1}} J(g(z))<\mu_{k}(m)+\varepsilon$. Set $\tilde{g}(z):=g(z) /$ $\left(\int_{\Omega} m^{\prime}|g(z)|^{p} d x\right)^{1 / p}$ for $z \in S^{k-1}$ (note $\int_{\Omega} m^{\prime}|g(z)|^{p} d x \geq \int_{\Omega} m|g(z)|^{p} d x=1$ ), then $\tilde{g} \in$ $\bar{\mp}_{k}\left(m^{\prime}\right)$ holds. Therefore, by the definition of $\mu_{k}\left(m^{\prime}\right)$, we have

$$
\mu_{k}\left(m^{\prime}\right) \leq \max _{z \in S^{k-1}} J(\tilde{g}(z))=\max _{z \in S^{k-1}} \frac{J(g(z))}{\int_{\Omega} m^{\prime}|g(z)|^{p} d x} \leq \max _{z \in S^{k-1}} J(g(z))<\mu_{k}(m)+\varepsilon .
$$

because of $\int_{\Omega} m^{\prime}|g(z)|^{p} d x \geq \int_{\Omega} m|g(z)|^{p} d x=1$ for every $z \in S^{k-1}$. Since $\varepsilon>0$ is arbitrary, we obtain $\mu_{k}\left(m^{\prime}\right) \leq \mu_{k}(m)$.

(ii) Let $\lim _{n \rightarrow \infty} m_{n}=m$ in $L^{r}(\Omega)$ and fix an arbitrary $\varepsilon>0$. By the definition of $\mu_{k}(m)$, there exists a $g \in \mathcal{F}_{k}(m)$ such that $\max _{z \in S^{k-1}} J(g(z))<\mu_{k}(m)+\varepsilon / 2$. Since $g\left(S^{k-1}\right)$ is compact and $p r^{\prime}:=p r /(r-1) \leq p^{*}$, we set $M:=\max _{u \in g\left(S^{k-1}\right)}\|u\|_{p r^{\prime}}$. Then, due to Hölder's inequality and $m_{n} \rightarrow m$ in $L^{r}(\Omega)$, there exists an $n_{0} \in \mathbb{N}$ such that

$$
\int_{\Omega} m_{n}|u|^{p} d x=1+\int_{\Omega}\left(m_{n}-m\right)|u|^{p} d x \geq 1-\left\|m_{n}-m\right\|_{r} M^{p}>0
$$

for every $u \in g\left(S^{k-1}\right)$ and $n \geq n_{0}$. Therefore, by a similar argument to (i), we obtain

$$
\mu_{k}\left(m_{n}\right) \leq \max _{z \in S^{k-1}} \frac{J(g(z))}{\int_{\Omega} m_{n}|g(z)|^{p} d x} \leq \frac{\mu_{k}(m)+\varepsilon / 2}{1-\left\|m_{n}-m\right\|_{r} M^{p}}<\mu_{k}(m)+\varepsilon
$$


for sufficiently large $n$. Hence, $\lim \sup _{n \rightarrow \infty} \mu_{k}\left(m_{n}\right) \leq \mu_{k}(m)+\varepsilon$ follows. Since $\varepsilon>0$ is arbitrary, our conclusion is proved.

(iii) Let $\lim _{n \rightarrow \infty} m_{n}=m$ in $L^{r}(\Omega)$ and $\int_{\Omega} m d x \neq 0$. We fix an arbitrary $\varepsilon>0$. Due to our assertion (ii), there exists an $n_{1} \in \mathbb{N}$ such that $\mu_{k}\left(m_{n}\right) \leq \mu_{k}(m)+\varepsilon / 2$. For every $n \geq n_{1}$, by the definition of $\mu_{k}\left(m_{n}\right)$, we can take $g_{n} \in \mathcal{F}_{k}\left(m_{n}\right)$ satisfying $\max _{z \in S^{k-1}} J\left(g_{n}(z)\right)<\mu_{k}\left(m_{n}\right)+\varepsilon / 2$.

Here, we will prove

$$
\sup _{n \geq n_{1}} \max \left\{\|u\|_{p} ; u \in g_{n}\left(S^{k-1}\right)\right\}<\infty
$$

If $u \in g_{n}\left(S^{k-1}\right)$ satisfies $\int_{\Omega} m|u|^{p} d x \leq 0$, then we obtain

$$
\begin{aligned}
b(m, 1)\|u\|_{p}^{p} & \leq J(u)-\int_{\Omega} m|u|^{p} d x=J(u)-\int_{\Omega} m_{n}|u|^{p} d x+\int_{\Omega}\left(m_{n}-m\right)|u|^{p} d x \\
& \leq \mu_{k}\left(m_{n}\right)+\frac{\varepsilon}{2}-1+\left\|m_{n}-m\right\|_{r}\|u\|_{p r^{\prime}}^{p} \\
& \leq \mu_{k}(m)+\varepsilon+C\left\|m_{n}-m\right\|_{r}\|u\|_{p}^{p}+\frac{C J(u)\left\|m_{n}-m\right\|_{r}}{\inf _{\Omega} a_{\infty}} \\
& \leq\left(1+\frac{C\left\|m_{n}-m\right\|_{r}}{\inf _{\Omega} a_{\infty}}\right)\left(\mu_{k}(m)+\varepsilon\right)+C\left\|m_{n}-m\right\|_{r}\|u\|_{p}^{p}
\end{aligned}
$$

by Lemma 2.2 and Hölder's inequality (note $\|\nabla u\|_{p}^{p} \leq J(u) / \inf _{\Omega} a_{\infty}$ and $\mu_{k}\left(m_{n}\right) \leq \mu_{k}(m)+$ $\varepsilon / 2$ ), where $C>0$ is a constant (independent of $n$ and $u$ ) obtained by the continuity of $W^{1, p}(\Omega)$ into $L^{p r^{\prime}}(\Omega)$. Therefore, if we take an $n_{2} \geq n_{1}$ satisfying $C\left\|m_{n}-m\right\|_{r} \leq b(m, 1) / 2$ for every $n \geq n_{2}$, then we obtain

$$
\|u\|_{p}^{p} \leq \frac{2}{b(m, 1)}\left(1+\frac{b(m, 1)}{2 \inf _{\Omega} a_{\infty}}\right)\left(\mu_{k}(m)+\varepsilon\right)
$$

for every $u \in g_{n}\left(S^{k-1}\right)$ provided $\int_{\Omega} m|u|^{p} d x \leq 0$ and $n \geq n_{2}$. Similarly, in the case where $m$ changes sign, for every $u \in g_{n}\left(S^{k-1}\right)$ satisfying $\int_{\Omega} m|u|^{p} d x>0$, we have

$$
\begin{aligned}
b(-m, 1)\|u\|_{p}^{p} & \leq J(u)-\int_{\Omega}(-m)|u|^{p} d x \\
& \leq\left(1+\frac{C\left\|m_{n}-m\right\|_{r}}{\inf _{\Omega} a_{\infty}}\right)\left(\mu_{k}(m)+\varepsilon\right)+1+C\left\|m_{n}-m\right\|_{r}\|u\|_{p}^{p} .
\end{aligned}
$$

Hence, by taking a sufficiently large $n_{3} \geq n_{2}$, we get the inequality

$$
\|u\|_{p}^{p} \leq \frac{2}{b(-m, 1)}\left(1+\frac{b(-m, 1)}{2 \inf _{\Omega} a_{\infty}}\right)\left(\mu_{k}(m)+\varepsilon+1\right),
$$


for every $u \in g_{n}\left(S^{k-1}\right)$ with $\int_{\Omega} m|u|^{p} d x>0$ and $n \geq n_{3}$. In the case of $m \geq 0$ in $\Omega$, by using Lemma 2.3 instead of Lemma 2.2, we have a similar inequality

$$
\|u\|_{p}^{p} \leq \frac{2}{d(m, 1)}\left(1+\frac{d(m, 1)}{2 \inf _{\Omega} a_{\infty}}\right)\left(\mu_{k}(m)+\varepsilon+1\right)
$$

for every $u \in g_{n}\left(S^{k-1}\right)$ provided $n \geq n_{4}$ (some sufficiently large $n_{4} \geq n_{3}$ ). Consequently, our claim follows from (2.15), (2.17), and (2.18).

Let us return to the proof of (iii). Because

$$
\sup \left\{\|u\|_{p r} ; u \in g_{n}\left(S^{k-1}\right), n \geq n_{1}\right\}=: R<+\infty
$$

holds by (2.13), $J(u) \leq \mu_{k}(m)+\varepsilon / 2$ and the continuity of $W^{1, p}(\Omega)$ into $L^{p r^{\prime}}(\Omega)$, we see the inequality

$$
\int_{\Omega} m|u|^{p} d x=1-\int_{\Omega}\left(m_{n}-m\right)|u|^{p} d x>1-\left\|m_{n}-m\right\|_{r} R^{p}>0,
$$

for every $u \in g_{n}\left(S^{k-1}\right)$ and $n \geq n_{5}$ (some sufficiently large $n_{5} \geq n_{4}$ ). By considering $\tilde{g}_{n}(\cdot):=$ $g_{n}(\cdot) /\left(\int_{\Omega} m\left|g_{n}(\cdot)\right|^{p} d x\right)^{1 / p} \in \mathcal{F}_{k}(m)$, we obtain

$$
\mu_{k}(m) \leq \max _{z \in S^{k-1}} J\left(\widetilde{g}_{n}(z)\right) \leq \frac{\max _{z \in S^{k-1}} J\left(g_{n}(z)\right)}{1-\left\|m_{n}-m\right\|_{r} R^{p}} \leq \frac{\mu_{k}\left(m_{n}\right)+\varepsilon / 2}{1-\left\|m_{n}-m\right\|_{r} R^{p}}
$$

Because of $\left\|m_{n}-m\right\|_{r} \rightarrow 0$, we get $\mu_{k}\left(m_{n}\right) \geq \mu_{k}(m)-\varepsilon$ for sufficiently large $n$, and hence our conclusion holds. theorem.

Finally, we recall the second eigenvalue of $(E V ; m)$ obtained by the mountain pass

$$
\begin{aligned}
\Sigma(m) & :=\{\eta \in C([0,1], S(m)) ; \eta(0) \in P, \eta(1) \in(-P)\}, \\
c(m) & :=\inf _{\eta \in \Sigma(m)} \max _{t \in[0,1]} \widetilde{J}(\eta(t)),
\end{aligned}
$$

where $P:=\left\{u \in W^{1, p}(\Omega) ; u(x) \geq 0\right.$ for a.e. $\left.x \in \Omega\right\}$.

Since $\infty>\sup _{x \in \Omega} a_{\infty}(x) \geq \inf _{x \in \Omega} a_{\infty}(x)>0$ holds, the following result can be shown by the same argument as in [19] (although they handle the asymmetry case, it is sufficient to consider the case of $m \equiv n$ in this paper). See [19, Theorem 3.2] for the proof.

Theorem 2.11. $c(m)$ is an eigenvalue of $(E V ; m)$ which satisfies $\lambda^{*}(m)<c(m)$. Moreover, there is no eigenvalues of $(E V ; m)$ between $\lambda^{*}(m)$ and $c(m)$. 
Now, we have the following result.

Proposition 2.12.

$$
\lambda_{2}(m)=\mu_{2}(m)=c(m)
$$

holds, where $c(m)$ is a minimax value defined by (2.22).

Proof. First, we prove the inequality $c(m) \geq \mu_{2}(m)$. Because $c(m)$ is an eigenvalue (note that the following equation is homogeneous), we can choose a solution $u \in W^{1, p}(\Omega)$ with $\int_{\Omega} m|u|^{p} d x=1$ for

$$
-\operatorname{div}\left(a_{\infty}(x)|\nabla u|^{p-2} \nabla u\right)=c(m) m(x)|u|^{p-2} u \quad \text { in } \Omega, \quad \frac{\partial u}{\partial v}=0 \quad \text { on } \partial \Omega .
$$

Note that $u$ is a sign-changing function because any eigenfunction associated with any eigenvalue greater than the principal eigenvalue changes sign (refer to [18, Proposition 4.3]). Thus, we have

$$
0<\int_{\Omega} a_{\infty}\left|\nabla u_{ \pm}\right|^{p} d x=c(m) \int_{\Omega} m u_{ \pm}^{p} d x
$$

by taking $\pm u_{ \pm}$as test function (recall that $u_{ \pm}:=\max \{ \pm u, 0\}$ ). Hence, we may assume that $\int_{\Omega} m u_{ \pm}^{p} d x=1$ by the normalization. Set $X:=\left\{s u_{+}-t u_{-} ;|s|^{p}+|t|^{p}=1\right\} \subset S(m)$. Then, because $X$ is homeomorphic to $S^{1}$, there exists $g \in \mathcal{F}_{2}(m)$ such that $g\left(S^{1}\right)=X$. Since the value of $J$ is equal to $c(m)$ on $X$, we obtain

$$
\mu_{2}(m) \leq \max _{z \in S^{1}} \widetilde{J}(g(z))=c(m)
$$

by the definition of $\mu_{2}(m)$ and $X$.

Next, we will prove the inequality $c(m) \leq \mathcal{\lambda}_{2}(m)$ by dividing into two cases: $\int_{\Omega} m d x \neq 0$ and $\int_{\Omega} m d x=0$.

Case of $\int_{\Omega} m d x \neq 0$ : by way of contradiction, we assume that $\lambda_{2}(m)<c(m)$. Then, $\lambda^{*}(m)=\lambda_{1}(m)=\lambda_{2}(m)$ follows from Theorem 2.11. Note that $\tilde{J}$ satisfies the Palais-Smale condition in this case (see Lemma 2.8), and hence we can apply the first deformation lemma to $\tilde{J}$. Therefore, by the standard argument (cf. [22], [17, Lemma 5.6]), we see that $\gamma(K) \geq 2$, where $K:=\left\{u \in S(m) ; \widetilde{J}^{\prime}(u)=0, \widetilde{J}(u)=\lambda^{*}(m)\right\}$. This means that $K$ is an infinite set, that is, the following equation has infinite many solutions:

$$
-\operatorname{div}\left(a_{\infty}(x)|\nabla u|^{p-2} \nabla u\right)=\lambda^{*}(m) m(x)|u|^{p-2} u \quad \text { in } \Omega, \quad \frac{\partial u}{\partial v}=0 \quad \text { on } \partial \Omega
$$

due to the Lagrange multiplier's rule. This contradicts to the fact described as in Proposition 2.4 that $\lambda^{*}(m)$ is simple. As a result, we have shown that $c(m)=\lambda_{2}(m)=\mu_{2}(m)$ holds in the case of $\int_{\Omega} m d x \neq 0$ (note $\left.\lambda_{n}(m) \leq \mu_{n}(m)\right)$. 
Case of $\int_{\Omega} m d x=0$ : According to Proposition 2.10 (i) for $\lambda_{2}(m)$, we have $\lambda_{2}(m) \geq$ $\lambda_{2}(m+\varepsilon)=c(m+\varepsilon)$ for every $\varepsilon>0$ since we can apply the first result to $m+\varepsilon$. Because we prove $\lim _{\varepsilon \rightarrow 0+} c(m+\varepsilon)=c(m)$ by the same argument as in [6, Lemma 2.9] (for the case $\left.a_{\infty} \equiv 1\right)$, our conclusion is proved by taking $\varepsilon \downarrow 0$ in the inequality $\lambda_{2}(m) \geq c(m+\varepsilon)$.

\section{Proof of Theorem 1.1}

We define a functional $I_{\lambda, m}$ on $W^{1, p}(\Omega)$ as follows:

$$
\begin{aligned}
I_{\lambda, m}(u)= & \int_{\Omega} G(x, \nabla u) d x-\frac{\lambda}{p} \int_{\Omega} m|u|^{p} d x-\int_{\Omega} F(x, u) d x-\int_{\Omega} h u d x \\
= & \frac{1}{p} \int_{\Omega} a_{\infty}|\nabla u|^{p} d x+\int_{\Omega} \tilde{G}(x, \nabla u) d x-\frac{\lambda}{p} \int_{\Omega} m|u|^{p} d x \\
& -\int_{\Omega} F(x, u) d x-\int_{\Omega} h u d x
\end{aligned}
$$

for $u \in W^{1, p}(\Omega)((1.15)$ or (1.9) for the definition of $G, \widetilde{G}$, and $F)$. It is easily seen that $I_{\lambda, m}$ is well defined and class of $C^{1}$ on $W^{1, p}(\Omega)$ by $(1.1),(1.16)$ and the continuity of $W^{1, p}(\Omega) \hookrightarrow$ $L^{p}(\Omega)$.

Remark 3.1. Let $u \in W^{1, p}(\Omega)$ be a critical point of $I_{\lambda, m}$, namely, $u$ satisfies the equality

$$
\int_{\Omega} A(x, \nabla u) \nabla \varphi d x=\lambda \int_{\Omega} m|u|^{p-2} u \varphi d x+\int_{\Omega} f(x, u) \varphi d x+\int_{\Omega} h \varphi d x
$$

for every $\varphi \in W^{1, p}(\Omega)$. Then, $u \in L^{\infty}(\Omega)$ by the Moser iteration process (refer to Theorem $C$ in [4]). Therefore, $u \in C^{1, \alpha}(\bar{\Omega})(0<\alpha<1)$ follows from the regularity result in [23]. Furthermore, due to [24, Theorem 3], $u$ satisfies $(P ; \lambda, m, h)$ in the distribution sense and the boundary condition

$$
0=\frac{\partial u}{\partial v_{A}}=A(\cdot, \nabla u) v=a(\cdot,|\nabla u|) \frac{\partial u}{\partial v} \quad \text { in } W^{-1 / q, q}(\partial \Omega)
$$

for every $1<q<\infty$ (see [24] for the definition of $W^{-1 / q, q}(\partial \Omega)$ ). Since $u \in C^{1, \alpha}(\bar{\Omega})$ and $a(x, t)>0$ for every $t \neq 0, u$ satisfies the Neumann boundary condition, that is, $(\partial u / \partial v)(x)=0$ for every $x \in \partial \Omega$.

\subsection{The Palais-Smale Condition in the Nonresonant Case}

First, we recall the definition of the Palais-Smale condition.

Definition 3.2. A $C^{1}$ functional $\Psi$ on a Banach space $X$ is said to satisfy the Palais-Smale condition at $c \in \mathbb{R}$ if a Palais-Smale sequence $\left\{u_{n}\right\} \subset X$ at level $c$, namely,

$$
\Psi\left(u_{n}\right) \longrightarrow c, \quad\left\|\Psi^{\prime}\left(u_{n}\right)\right\|_{X^{*}} \longrightarrow 0 \quad \text { as } n \longrightarrow \infty
$$


has a convergent subsequence. We say that $\Psi$ satisfies the Palais-Smale condition if $\Psi$ satisfies the Palais-Smale condition at any $c \in \mathbb{R}$. Moreover, we say that $\Psi$ satisfies the bounded Palais-Smale condition if any bounded sequence $\left\{u_{n}\right\}$ such that $\left\{\Psi\left(u_{n}\right)\right\}$ is bounded and $\left\|\Psi^{\prime}\left(u_{n}\right)\right\|_{X^{*}} \rightarrow 0$ as $n \rightarrow \infty$ has a convergent subsequence.

Concerning the Palais-Smale condition, we state the following result developed from [6, Proposition 7].

Proposition 3.3. If $\lambda$ is not an eigenvalue of $(E V ; m)$, then $I_{\lambda, m}$ satisfies the Palais-Smale condition.

Proof. Let $\left\{u_{n}\right\}$ be a Palais-Smale sequence of $I_{\lambda, m}$, namely,

$$
I_{\lambda, m}\left(u_{n}\right) \longrightarrow c, \quad\left\|I_{\lambda, m}^{\prime}\left(u_{n}\right)\right\|_{W^{1, p}(\Omega)^{*}} \longrightarrow 0 \quad \text { as } n \longrightarrow \infty
$$

for some $c \in \mathbb{R}$. It is sufficient to prove only the boundedness of $\left\|u_{n}\right\|$ because the operator $A: W^{1, p}(\Omega) \rightarrow W^{1, p}(\Omega)^{*}$ described in Proposition 1.5 has the $(S)_{+}$property.

To prove the boundedness of $\left\|u_{n}\right\|$, it suffices to show that $\left\|u_{n}\right\|_{p}$ is bounded because of the inequality $|f(x, u)| \leq C\left(|u|^{p-1}+1\right)$ (obtained by (1.1)) and the following inequality:

$$
\begin{gathered}
\left\langle I_{\lambda, m}^{\prime}\left(u_{n}\right), u_{n}\right\rangle+\lambda \int_{\Omega} m\left|u_{n}\right|^{p} d x+\int_{\Omega} f\left(x, u_{n}\right) u_{n} d x+\int_{\Omega} h u_{n} d x, \\
=\int_{\Omega} A\left(x, \nabla u_{n}\right) \nabla u_{n} d x \geq \frac{C_{0}}{p-1}\left\|\nabla u_{n}\right\|_{p}^{p}
\end{gathered}
$$

where we use Remark 1.4 (iii) in the last inequality. By way of contradiction, we may assume that $\left\|u_{n}\right\|_{p} \rightarrow \infty$ as $n \rightarrow \infty$ by choosing a subsequence if necessary. Set $v_{n}:=u_{n} /\left\|u_{n}\right\|_{p}$. Then, since the inequality (3.6) guarantees that $\left\{v_{n}\right\}$ is bounded in $W^{1, p}(\Omega)$, we may suppose, by choosing a subsequence, that $v_{n} \rightarrow v_{0}$ in $W^{1, p}(\Omega)$ and $v_{n} \rightarrow v_{0}$ in $L^{p}(\Omega)$ for some $v_{0}$.

Here, we will prove that

$$
\lim _{n \rightarrow \infty} \frac{\left\|f\left(\cdot, u_{n}\right)\right\|_{p^{\prime}}}{\left\|u_{n}\right\|_{p}^{p-1}}=0,
$$

where $p^{\prime}=p /(p-1)$. Fix an arbitrary $\varepsilon>0$. It follows from (1.1) that there exists a $C_{\varepsilon}>0$ such that

$$
|f(x, u)| \leq \varepsilon|u|^{p-1}+C_{\varepsilon} \quad \text { for every } u \in \mathbb{R}, \quad \text { a.e. } x \in \Omega
$$

Then, we obtain

$$
\int_{\Omega}\left|f\left(x, u_{n}\right)\right|^{p^{\prime}} d x \leq 2^{p^{\prime}} \int_{\Omega}\left(\varepsilon^{p^{\prime}}\left|u_{n}\right|^{p}+C_{\varepsilon}^{p^{\prime}}\right) d x \leq 2^{p^{\prime}} \varepsilon^{p^{\prime}}\left\|u_{n}\right\|_{p}^{p}+2^{p^{\prime}} C_{\varepsilon}^{p^{\prime}}|\Omega|
$$


Since we are assuming that $\left\|u_{n}\right\|_{p} \rightarrow \infty$ as $n \rightarrow \infty$, there exists $n_{0} \in \mathbb{N}$ such that for every $n \geq n_{0}$

$$
\frac{\left\|f\left(\cdot, u_{n}\right)\right\|_{p^{\prime}}}{\left\|u_{n}\right\|_{p}^{p-1}} \leq 4 \varepsilon
$$

holds. This shows that $\lim _{n \rightarrow \infty}\left\|f\left(\cdot, u_{n}\right)\right\|_{p^{\prime}} /\left\|u_{n}\right\|_{p}^{p-1}=0$ because $\varepsilon>0$ is arbitrary.

Here, we recall the following result proved in [6]:

$$
\lim _{n \rightarrow \infty} \int_{\Omega} \frac{\tilde{a}\left(x,\left|\nabla u_{n}\right|\right) \nabla u_{n}}{\left\|u_{n}\right\|_{p}^{p-1}} \nabla\left(v_{n}-v_{0}\right) d x=\lim _{n \rightarrow \infty} \int_{\Omega} \frac{\tilde{a}\left(x,\left|\nabla u_{n}\right|\right) \nabla u_{n}}{\left\|u_{n}\right\|_{p}^{p-1}} \nabla \varphi d x=0,
$$

for every $\varphi \in W^{1, p}(\Omega)$. Thus, by considering

$$
o(1)=\frac{\left\langle I_{\lambda, m}^{\prime}\left(u_{n}\right), v_{n}-v_{0}\right\rangle}{\left\|u_{n}\right\|_{p}^{p-1}}=\int_{\Omega} a_{\infty}\left|\nabla v_{n}\right|^{p-2} \nabla v_{n} \nabla\left(v_{n}-v_{0}\right) d x+o(1)
$$

we see that $v_{n}$ strongly converges to $v_{0}$ in $W^{1, p}(\Omega)$ (note that $p$-Laplacian has the $(S)_{+}$property). Therefore, by taking a limit in $o(1)=\left\langle I_{\lambda, m}^{\prime}\left(u_{n}\right), \varphi\right\rangle /\left\|u_{n}\right\|_{p}^{p-1}$ for any $\varphi \in W^{1, p}(\Omega)$ and by noting (3.7) and (3.11), we know that $v_{0}$ is a nontrivial solution (note $\left\|v_{0}\right\|_{p}=1$ ) of

$$
-\operatorname{div}\left(a_{\infty}|\nabla u|^{p-2} \nabla u\right)=\lambda m|u|^{p-2} u \quad \text { in } \Omega, \quad \frac{\partial u}{\partial v}=0 \quad \text { on } \partial \Omega
$$

This means that $\lambda$ is an eigenvalue of $(E V ; m)$. This is a contradiction. Hence, $\left\|u_{n}\right\|_{p}$ is bounded.

\subsection{Key Lemmas}

To show the linking lemma, we define

$$
Y(\mu, m):=\left\{u \in W^{1, p}(\Omega) ; \int_{\Omega} a_{\infty}|\nabla u|^{p} d x \geq \mu \int_{\Omega} m|u|^{p} d x\right\}
$$

for $\mu \in \mathbb{R}$.

Lemma 3.4. Let $g_{0} \in C\left(S^{k-1}, W^{1, p}(\Omega) \backslash\{0\}\right)$ be odd and $0<\mu \leq \mu_{k+1}(m)$. Then, $g\left(S_{+}^{k}\right) \cap$ $\Upsilon(\mu, m) \neq \emptyset$ for every $g \in C\left(S_{+}^{k}, W^{1, p}(\Omega)\right)$ with $\left.g\right|_{S^{k-1}}=g_{0}$, where $Y(\mu, m)$ is the set introduced in (3.14) and $S_{+}^{k}$ is the upper hemisphere in $\mathbb{R}^{k+1}$ with boundary $S^{k-1}$. 
Proof. Fix any $g \in C\left(S_{+}^{k}, W^{1, p}(\Omega)\right)$ such that $\left.g\right|_{S^{k-1}}=g_{0}$. If $u \in g\left(S_{+}^{k}\right)$ satisfies $\int_{\Omega} m|u|^{p} d x \leq 0$, then $u \in Y(\mu, m)$ holds. So, we may assume that $\int_{\Omega} m|u|^{p} d x>0$ for every $u \in g\left(S_{+}^{k}\right)$. Define $\tilde{g} \in \mathcal{F}_{k+1}(m)$ as follows:

$$
\tilde{g}(z):= \begin{cases}\frac{g(z)}{\left(\int_{\Omega} m|g(z)|^{p} d x\right)^{1 / p}} & \text { if } z \in S_{+}^{k}, \\ -\frac{g(-z)}{\left(\int_{\Omega} m|g(-z)|^{p} d x\right)^{1 / p}} & \text { if } z \in S_{-}^{k} .\end{cases}
$$

By the definition of $\mu_{k+1}(m)$, there exists $z_{0} \in S^{k}$ such that $\widetilde{J}\left(\widetilde{g}\left(z_{0}\right)\right) \geq \mu_{k+1}(m)$. Since $\tilde{g}$ is odd and $J$ is even, we may suppose $z_{0} \in S_{+}^{k}$. So, this yields the inequality $J\left(g\left(z_{0}\right)\right) \geq$ $\mu_{k+1}(m) \int_{\Omega} m\left|g\left(z_{0}\right)\right|^{p} d x \geq \mu \int_{\Omega} m\left|g\left(z_{0}\right)\right|^{p} d x$, whence $g\left(z_{0}\right) \in Y(\mu, m)$ holds.

Lemma 3.5. Let $\mu_{k}(m)<\lambda$. Then, there exists $g_{0} \in \mathcal{F}_{k}(m)$ such that

$$
\max _{z \in S^{k-1}} J\left(g_{0}(z)\right)<\lambda, \quad \max _{z \in S^{k-1}} I_{\lambda, m}\left(T g_{0}(z)\right) \longrightarrow-\infty \quad \text { as }|T| \longrightarrow \infty
$$

where $\mu_{k}(m)$ is defined by (2.5).

Proof. Choose $\varepsilon_{0}>0$ such that $\mu_{k}(m)+\varepsilon_{0}<\lambda$. By the definition of $\mu_{k}(m)$, there exists $g_{0} \in$ $\mathcal{F}_{k}(m)$ such that

$$
\max _{z \in S^{k-1}} J\left(g_{0}(z)\right)<\mu_{k}(m)+\varepsilon_{0} .
$$

Due to the compactness of $g_{0}\left(S^{k-1}\right)$, we put $M:=\max _{z \in S^{k-1}}\left\|g_{0}(z)\right\|_{p}$. By the property of the function $\tilde{a}$ as in $(A H)$ and Young's inequality, for every $\varepsilon>0$ there exist constants $C_{\varepsilon}>0$ and $C_{\varepsilon}^{\prime}>0$ such that

$$
|\widetilde{G}(x, y)| \leq \frac{\varepsilon}{2}|y|^{p}+C_{\varepsilon}|y| \leq \varepsilon|y|^{p}+C_{\varepsilon}^{\prime} \leq \frac{\varepsilon}{\inf _{\Omega} a_{\infty}} a_{\infty}(x)|y|^{p}+C_{\varepsilon}^{\prime}
$$

for every $x \in \Omega$ and $y \in \mathbb{R}^{N}$. Moreover, the hypothesis (1.1) ensures that for every $\varepsilon^{\prime}>0$ there exist constants $D_{\varepsilon^{\prime}}>0$ satisfying

$$
|F(x, u)| \leq \frac{\varepsilon^{\prime}}{2}|u|^{p}+D_{\varepsilon^{\prime}}|u| \leq \varepsilon^{\prime}|u|^{p}+D_{\varepsilon^{\prime}}^{\prime}
$$

for every $u \in \mathbb{R}$ and a.e. $x \in \Omega$. Hence, we have

$$
\begin{aligned}
I_{\lambda, m}(T u) & \leq \frac{T^{p}}{p}\left(1+\frac{p \varepsilon}{\underline{a}}\right) \int_{\Omega} a_{\infty}|\nabla u|^{p} d x-\frac{T^{p}\left(\lambda-p \varepsilon^{\prime} M^{p}\right)}{p}+T\|h\|_{\infty}\|u\|_{1}+C \\
& \leq \frac{T^{p}}{p}\left\{\left(1+\frac{p \varepsilon}{\underline{a}}\right)\left(\mu_{k}(m)+\varepsilon_{0}\right)-\lambda+p M^{p} \varepsilon^{\prime}\right\}+T M\|h\|_{\infty}|\Omega|^{(p-1) / p}+C
\end{aligned}
$$


for every $T>0, u \in g_{0}\left(S^{k-1}\right), \varepsilon>0$ and $\varepsilon^{\prime}>0$ since $g_{0}\left(S^{k-1}\right) \subset S(m),(3.17)$, (3.18) and (3.19), where $C=\left(C_{\varepsilon}^{\prime}+D_{\varepsilon^{\prime}}^{\prime}\right)|\Omega|$ and $\underline{a}=\inf _{x \in \Omega} a_{\infty}(x)>0$. By taking $\varepsilon>0$ and $\varepsilon^{\prime}>0$ satisfying $(1+p \varepsilon / \underline{a})\left(\mu_{k}(m)+\varepsilon_{0}\right)-\lambda+p M^{p} \varepsilon^{\prime}<0$, we show that $\max _{z \in S^{k-1}} I_{\lambda, m}\left(T g_{0}(z)\right) \rightarrow-\infty$ as $T \rightarrow+\infty$. Thus, our conclusion follows because $g_{0}\left(S^{k-1}\right)$ is symmetric.

\subsection{The Case $\int_{\Omega} m d x \neq 0$}

Lemma 3.6. Let $\int_{\Omega} m d x<0$ and $0<\lambda<\lambda^{*}(m)$. Then, $I_{\lambda, m}$ is bounded from below, coercive and weakly lower semicontinuous (w.l.s.c.) on $W^{1, p}(\Omega)$.

Proof. $\Phi(u):=\int_{\Omega} G(x, \nabla u) d x$ is w.l.s.c. on $W^{1, p}(\Omega)$ because $\Phi$ is convex and continuous on $W^{1, p}(\Omega)$ (cf. [25, Theorem 1.2]). Thus, $I_{\lambda, m}$ is also w.l.s.c. on $W^{1, p}(\Omega)$ since the inclusion from $W^{1, p}(\Omega)$ to $L^{p}(\Omega)$ is compact.

Choose $\varepsilon>0$ such that $p \varepsilon<\underline{a}\left(1-\lambda / \lambda^{*}(m)\right)$, where $\underline{a}:=\inf _{\Omega} a_{\infty}$. By an easy estimation, (3.18) and (3.19) as in Lemma 3.5, we have

$$
\begin{aligned}
I_{\lambda, m}(u) \geq & \frac{\underline{a}-\varepsilon p}{p \underline{a}} \int_{\Omega} a_{\infty}|\nabla u|^{p} d x-\frac{\lambda}{p} \int_{\Omega} m|u|^{p} d x-\varepsilon^{\prime}\|u\|_{p}^{p} \\
& -\|h\|_{\infty}\|u\|_{p}|\Omega|^{(p-1) / p}-\left(C_{\varepsilon}^{\prime}+D_{\varepsilon^{\prime}}^{\prime}\right)|\Omega|
\end{aligned}
$$

for every $u \in W^{1, p}(\Omega)$ and $\varepsilon^{\prime}>0$.

Let $u \in W^{1, p}(\Omega)$ satisfy $\int_{\Omega} m|u|^{p} d x \leq 0$. Then, the following inequality follows from Lemma 2.2:

$$
D_{0} \int_{\Omega} a_{\infty}|\nabla u|^{p} d x-\lambda \int_{\Omega} m|u|^{p} d x \geq \frac{D_{0}}{2} \int_{\Omega} a_{\infty}|\nabla u|^{p} d x+b(m, \xi)\|u\|_{p}^{p}
$$

where $b(m, \xi)$ is a positive constant independent of $u$ with $\xi=2 \lambda / D_{0}$ and $D_{0}=(\underline{a}-\varepsilon p) / \underline{a}$.

For every $u \in W^{1, p}(\Omega)$ such that $\int_{\Omega} m|u|^{p} d x>0$, we obtain

$$
\begin{gathered}
D_{0} \int_{\Omega} a_{\infty}|\nabla u|^{p} d x-\lambda \int_{\Omega} m|u|^{p} d x \geq\left(D_{0}-\frac{\lambda}{\lambda^{*}(m)}\right) \int_{\Omega} a_{\infty}|\nabla u|^{p} d x \\
\geq \frac{1}{2}\left(D_{0}-\frac{\lambda}{\lambda^{*}(m)}\right) \int_{\Omega} a_{\infty}|\nabla u|^{p} d x+\frac{c}{2}\left(D_{0}-\frac{\lambda}{\lambda^{*}(m)}\right)\|u\|_{p}^{p}
\end{gathered}
$$

by the definition of $\lambda^{*}(m)$, Lemma 2.1 and $D_{0}-\lambda / \lambda^{*}(m)>0$, where $c>0$ is a constant obtained by Lemma 2.1.

Consequently, if we choose a $\varepsilon^{\prime}>0$ satisfying $\varepsilon^{\prime}<\min \left\{b(m, \xi) / p, c\left(D_{0}-\lambda / \lambda^{*}(m)\right) /\right.$ $(2 p)\}$, then we obtain positive constants $d_{1}$ and $d_{2}$ (independent of $u$ ) such that

$$
\begin{aligned}
I_{\lambda, m}(u) & \geq d_{1} \int_{\Omega} a_{\infty}|\nabla u|^{p} d x+d_{2}\|u\|_{p}^{p}-\|h\|_{\infty}\|u\|_{p}|\Omega|^{(p-1) / p}-\left(C_{\varepsilon}^{\prime}+D_{\varepsilon^{\prime}}^{\prime}\right)|\Omega| \\
& \geq \min \left\{\underline{a} d_{1}, d_{2}\right\}\|u\|^{p}-\|h\|_{\infty}\|u\||\Omega|^{(p-1) / p}-\left(C_{\varepsilon}^{\prime}+D_{\varepsilon^{\prime}}^{\prime}\right)|\Omega|
\end{aligned}
$$


for every $u \in W^{1, p}(\Omega)$ by (3.21), (3.22), and (3.23). Because of $p>1$, our conclusion is shown.

Lemma 3.7. Let $m \geq 0$ in $\Omega$ and $m \neq \equiv$. If $\lambda<0$ holds, then $I_{\lambda, m}$ is bounded from below, coercive and w.l.s.c. on $W^{1, p}(\Omega)$.

Proof. First, as the same reason in Lemma 3.6, it follows that $I_{\lambda, m}$ is w.l.s.c. on $W^{1, p}(\Omega)$. By a similar argument to Lemma 3.6, for every $\varepsilon^{\prime}>0$ and $0<\varepsilon<\underline{a} / p$ where $\underline{a}=\inf _{\Omega} a_{\infty}$, we obtain

$$
\begin{aligned}
I_{\lambda, m}(u) \geq & \frac{\underline{a}-\varepsilon p}{p \underline{a}} \int_{\Omega} a_{\infty}|\nabla u|^{p} d x+\frac{|\lambda|}{p} \int_{\Omega} m|u|^{p} d x-\varepsilon^{\prime}\|u\|_{p}^{p} \\
& -\|h\|_{\infty}\|u\|_{p}|\Omega|^{(p-1) / p}-\left(C_{\varepsilon}^{\prime}+D_{\varepsilon^{\prime}}^{\prime}\right)|\Omega|
\end{aligned}
$$

for every $u \in W^{1, p}(\Omega)$ (note $\lambda<0$ ). Here, from Lemma 2.3,

$$
D_{0} \int_{\Omega} a_{\infty}|\nabla u|^{p} d x+|\lambda| \int_{\Omega} m|u|^{p} d x \geq \frac{D_{0}}{2} \int_{\Omega} a_{\infty}|\nabla u|^{p} d x+\frac{D_{0}}{2} b(\xi, m)\|u\|_{p}^{p}
$$

for every $u \in W^{1, p}(\Omega)$ follows, where $D_{0}:=(\underline{a}-\varepsilon p) / \underline{a}, \xi:=2|\lambda| / D_{0}$ and $b(\xi, m)$ is a constant obtained in Lemma 2.3. Therefore, by choosing a $\varepsilon^{\prime}$ such that $0<\varepsilon^{\prime}<D_{0} b(\xi, m) / 2$, we can prove our conclusion.

Lemma 3.8. Let $\int_{\Omega} m d x \neq 0$ and $0<\lambda<\mu$. Then, $I_{\lambda, m}$ is bounded from below on $Y(\mu, m)$, where $Y(\mu, m)$ is the set introduced in (3.14).

Proof. Due to the same inequalities concerning $G$ and $F$ as in Lemma 3.5, for every $\varepsilon>0$ and $\varepsilon^{\prime}>0$, there exists $C=C\left(\varepsilon, \varepsilon^{\prime}\right)>0$ such that

$$
I_{\lambda, m}(u) \geq \frac{\underline{a}-p \varepsilon}{p \underline{a}} \int_{\Omega} a_{\infty}|\nabla u|^{p} d x-\frac{\lambda}{p} \int_{\Omega} m|u|^{p} d x-\varepsilon^{\prime}\|u\|_{p}^{p}-\|h\|_{\infty}\|u\|_{1}-C|\Omega|
$$

for every $u \in W^{1, p}(\Omega)$, where $\underline{a}:=\inf _{x \in \Omega} a_{\infty}(x)$. Choose positive constants $\varepsilon$ and $\delta$ such that $D_{0}:=1-p \varepsilon / \underline{a}>\delta>\lambda / \mu($ note $\lambda / \mu<1)$.

First, we consider the case of $m \geq 0$ in $\Omega$. For every $u \in Y(\mu, m)$, we obtain

$$
\begin{aligned}
& D_{0} \int_{\Omega} a_{\infty}|\nabla u|^{p} d x-\lambda \int_{\Omega} m|u|^{p} d x \\
& \quad \geq\left(D_{0}-\delta\right) \int_{\Omega} a_{\infty}|\nabla u|^{p} d x+(\delta \mu-\lambda) \int_{\Omega} m|u|^{p} d x \geq d\left(m, \xi_{1}\right)\left(D_{0}-\delta\right)\|u\|_{p}^{p}
\end{aligned}
$$

by Lemma 2.3 with $\xi_{1}=(\delta \mu-\lambda) /\left(D_{0}-\delta\right)$ (note $\delta \mu-\lambda>0$ and $\left.D_{0}-\delta>0\right)$. 
Next, we handle with the case where $m$ changes sign. Let $u \in W^{1, p}(\Omega)$ satisfy $\int_{\Omega} m|u|^{p} d x \leq 0$. Then, we have for such $u$

$$
D_{0} \int_{\Omega} a_{\infty}|\nabla u|^{p} d x-\lambda \int_{\Omega} m|u|^{p} d x \geq b\left(m, \xi_{2}\right) D_{0}\|u\|_{p}^{p}
$$

by Lemma 2.2, where $D_{0}=1-p \varepsilon / \underline{a}$ and $\xi_{2}:=\lambda / D_{0}$.

On the other hand, for $u \in \bar{Y}(\mu, m)$ with $\int_{\Omega} m|u|^{p} d x>0$, the following inequality follows from Lemma 2.2:

$$
\begin{aligned}
& D_{0} \int_{\Omega} a_{\infty}|\nabla u|^{p} d x-\lambda \int_{\Omega} m|u|^{p} d x \\
& \quad \geq\left(D_{0}-\delta\right) \int_{\Omega} a_{\infty}|\nabla u|^{p} d x-(\delta \mu-\lambda) \int_{\Omega}(-m)|u|^{p} d x \\
& \quad \geq b\left(-m, \xi_{1}\right)\left(D_{0}-\delta\right)\|u\|_{p}^{p} .
\end{aligned}
$$

Consequently, by (3.27), (3.29), (3.28), and (3.30), there exists $d>0$ independent of $u$ such that

$$
I_{\lambda, m}(u) \geq\left(d-\varepsilon^{\prime}\right)\|u\|_{p}^{p}-\|h\|_{\infty}\|u\|_{p}|\Omega|^{(p-1) / p}-C|\Omega|
$$

for every $u \in Y(\mu, m)$. Hence, our conclusion is shown by taking $\varepsilon^{\prime}>0$ satisfying $\varepsilon^{\prime}<d$.

Proof of Theorem 1.1 in the Case $\int_{\Omega} m d x \neq 0$. First, if either $m \geq 0$ on $\Omega$ and $\lambda<0$ or $0<\lambda<$ $\lambda^{*}(m)=\mu_{1}(m)$ (i.e., $\int_{\Omega} m d x<0$ ) holds, then Lemma 3.7 or Lemma 3.6 guarantees the existence of a global minimizer of $I_{\lambda, m}$, respectively (cf. [25, Theorem 1.1]). Hence, $(P ; \lambda, m, h)$ has a solution.

Since $\lambda$ is an eigenvalue of $(E V ; m)$ if and only if $-\lambda$ is one of $(E V ;-m)$, it suffices to consider the case of $\lambda>\lambda^{*}(m) \geq 0$. Furthermore, by Proposition 2.9, Remark 2.6 (i), and our hypothesis that $\lambda$ is not an eigenvalue of $(E V ; m)$, we may assume that there exists a $k \in \mathbb{N}$ such that $\mu_{k}(m)<\lambda<\mu_{k+1}(m)$. By Lemmas 3.5 and 3.8, we can choose $T>0$ and $g_{0} \in \mathscr{F}_{k}(m)$ satisfying

$$
\max _{z \in S^{k-1}} I_{\lambda, \mathrm{m}}\left(T g_{0}(z)\right)<\inf \left\{I_{\lambda, m}(u) ; u \in Y\left(\mu_{k+1}(m), m\right)\right\}=: \alpha
$$

Put

$$
\begin{gathered}
\Sigma:=\left\{g \in C\left(S_{+}^{k}, W^{1, p}(\Omega)\right) ;\left.g\right|_{S^{k-1}}=T g_{0}\right\}, \\
C:=\inf _{g \in \Sigma} \max _{z \in S_{+}^{k}} I_{\lambda, m}(g(z)) .
\end{gathered}
$$

Then, it follows from Lemma 3.4 and (3.32) that $c \geq \alpha>\max _{z \in S^{k-1}} I_{\lambda, m}\left(T g_{0}(z)\right)$ holds. Since $I_{\lambda, m}$ satisfies the Palais-Smale condition by Proposition 3.3, the minimax theorem guarantees (cf. [25, Theorem 4.6]) that $c$ is a critical value of $I_{\lambda, m}$. Hence, $(P ; \lambda, m, h)$ has at least one solution. 


\subsection{The Case $\int_{\Omega} m d x=0$}

First, we introduce an approximate functional $I_{\lambda, m, n}^{+}$as follows:

$$
I_{\lambda, m, n}^{+}(u):=I_{\lambda, m}(u)+\frac{1}{p n}\|u\|_{p}^{p}=I_{\lambda, m-1 /(\lambda n)}(u) \quad \text { for } u \in W^{1, p}(\Omega) .
$$

Lemma 3.9. Let $0<\lambda<\mu$. Then, there exists an $n_{0} \in \mathbb{N}$ such that for each $n \geq n_{0}, I_{\lambda, m, n}^{+}$is bounded from below on $Y(\mu, m-1 / \lambda n)$, where $Y(\mu, m-1 / \lambda n)$ is the set introduced in (3.14).

Proof. Choose $n_{0} \in \mathbb{N}$ such that $1 / n_{0}<\lambda$ ess $\sup _{x \in \Omega} m(x) / 2$. Then, for every $n \geq n_{0}$, Lemma 3.8 guarantees that $I_{\lambda, m, n}^{+}=I_{\lambda, m-1 /(\lambda n)}$ bounded from below on $Y(\mu, m-1 /(\lambda n))$ because of $\int_{\Omega}(m-1 /(\lambda n)) d x<0$ and $\left|\left\{m-1 /\left(\lambda_{n}\right)>0\right\}\right|>0$.

Proof of Theorem 1.1 in the Case $\int_{\Omega} m d x=0$. By noting that $\lambda m=(-\lambda)(-m)$ and $\mu_{1}(m)=$ $\lambda^{*}(m)=0$, we may assume that $\mu_{k}(m)<\lambda<\mu_{k+1}(m)$ for some $k \in \mathbb{N}$. Let $n_{0}$ be a natural number obtained by Lemma 3.9. Due to Proposition 2.10 (i) and (ii), there exists an $n_{1} \geq n_{0}$ such that

$$
\mu_{k}(m) \leq \mu_{k}\left(m-\frac{1}{n \lambda}\right) \leq \mu_{k}\left(m-\frac{1}{n_{1} \lambda}\right)<\lambda<\mu_{k+1}(m) \leq \mu_{k+1}\left(m-\frac{1}{n \lambda}\right)
$$

for every $n \geq n_{1}$. Thus, for every $n \geq n_{1}$, we can take $T_{n}>0$ and $g_{n} \in \mathcal{F}_{k}(m-1 /(n \lambda))$ satisfying

$$
\max _{z \in S^{k-1}} I_{\lambda, m, n}^{+}\left(T_{n} g_{n}(z)\right)<\inf \left\{I_{\lambda, m, n}(u) ; u \in \mathrm{Y}\left(\mu_{k+1}\left(m-\frac{1}{(n \lambda)}\right), m-\frac{1}{(n \lambda)}\right)\right\}
$$

by applying Lemmas 3.5 and 3.9 to $I_{\lambda, m, n}^{+}=I_{\lambda, m-1 /(n \lambda)}($ note (3.35)). Set

$$
\begin{gathered}
\Sigma_{n}:=\left\{g \in C\left(S_{+}^{k}, W^{1, p}(\Omega)\right) ;\left.g\right|_{S^{k-1}}=T_{n} g_{n}\right\}, \\
\mathcal{C}_{n}:=\inf _{g \in \Sigma_{n}} \max _{z \in S_{+}^{k}} I_{\lambda, m, n}^{+}(g(z))
\end{gathered}
$$

for each $n \geq n_{1}$. Then, for each $n \geq n_{1}$, we can obtain $u_{n}$ satisfying

$$
\left|I_{\lambda, m, n}^{+}\left(u_{n}\right)-c_{n}\right|<\frac{1}{n}, \quad\left\|\left(I_{\lambda, m, n}^{+}\right)^{\prime}\left(u_{n}\right)\right\|_{W^{1, p}(\Omega)}<\frac{1}{n}
$$

by applying Ekeland's variational principle to each $I_{\lambda, m, n}^{+}$(refer to [25, Theorem 4.3]). In addition, we can see that $\left\{u_{n}\right\}$ is bounded in $W^{1, p}(\Omega)$. Indeed, if there exists a subsequence $\left\{u_{n_{l}}\right\}_{l}$ satisfying $\left\|u_{n_{l}}\right\|_{p} \rightarrow \infty$ as $l \rightarrow \infty$, then we can show that $\lambda$ is an eigenvalue of $(E V ; m)$ by the same argument as in Proposition 3.3. This contradicts to our assumption that $\lambda$ is not an eigenvalue of $(E V ; m)$. Moreover, the boundedness of $\left\|\nabla u_{n}\right\|_{p}$ follows from a similar inequality to (3.6) as in Proposition 3.3 under the boundedness of $\left\|u_{n}\right\|_{p}$. 
Therefore, we may assume, by choosing a subsequence that $\left\{u_{n}\right\}$ is a Palais-Smale sequence of $I_{\lambda, m}$ since $I_{\lambda, m}$ is bounded on a bounded set and according to the following inequality:

$$
\left\|I_{\lambda, m}^{\prime}\left(u_{n}\right)\right\|_{\left(W^{1, p}(\Omega)\right)^{*}} \leq\left\|I_{\lambda, m}^{\prime}\left(u_{n}\right)-\left(I_{\lambda, m, n}^{+}\right)^{\prime}\left(u_{n}\right)\right\|_{\left(W^{1, p}(\Omega)\right)^{*}}+\frac{1}{n} \leq \frac{1}{n}\left\|u_{n}\right\|_{p}^{p-1}+\frac{1}{n} .
$$

Therefore, because $I_{\lambda, m}$ satisfies the Palais-Smale condition by Proposition 3.3, $I_{\lambda, m}$ has a critical point, whence $(P ; \lambda, m, h)$ has at least one solution.

\section{Proof of Theorem 1.2}

First, we will prove the following result concerning the Palais-Smale condition under the additional hypothesis $(H \pm)$ or $(H F \pm)$.

Proposition 4.1. Assume that one of the following conditions hold:

(i) $\lambda=0$ and $(H F+)$ or $(H F-)$;

(ii) $\lambda \neq 0$ and one of $(H+),(H-),(H F+)$ and $(H F-)$.

Then, $I_{\lambda, m}$ satisfies the Palais-Smale condition.

Proof. As the same reason in Proposition 3.3, it suffices to prove the boundedness of a PalaisSmale sequence $\left\{u_{n}\right\}$ such that $I_{\lambda, m}\left(u_{n}\right) \rightarrow c$ (for some $c \in \mathbb{R}$ ) and $\left\|I_{\lambda, m}^{\prime}\left(u_{n}\right)\right\|_{W^{*}} \rightarrow 0$ as $n \rightarrow \infty$. By way of contradiction, we may assume that $\left\|u_{n}\right\|_{p} \rightarrow \infty$ as $n \rightarrow \infty$ by choosing a subsequence. Set $v_{n}:=u_{n} /\left\|u_{n}\right\|_{p}$. Then, by the same argument as in Proposition 3.3, $\left\{v_{n}\right\}$ has a subsequence strongly convergent to $v_{0}$ being a nontrivial solution of

$$
-\operatorname{div}\left(a_{\infty}(x)|\nabla u|^{p-2} \nabla u\right)=\lambda m(x)|u|^{p-2} u \quad \text { in } \Omega, \quad \frac{\partial u}{\partial v}=0 \quad \text { on } \partial \Omega .
$$

To simplify the notation, we denote the above subsequence strongly convergent to $v_{0}$ by $\left\{v_{n}\right\}$, again. Thus, $\left|u_{n}(x)\right| \rightarrow \infty$ as $n \rightarrow \infty$ for a.e. $x \in \Omega_{0}:=\left\{x^{\prime} \in \Omega ; v_{0}\left(x^{\prime}\right) \neq 0\right\}$ (note $\left\|v_{0}\right\|_{p}=1$ ).

Assume $(H F+)$ or $(H F-)$. Then, we can obtain

$$
(I):=\int_{\Omega} \frac{f\left(x, u_{n}\right) u_{n}-p F\left(x, u_{n}\right)}{\left\|u_{n}\right\|_{p}^{1+q}} d x \longrightarrow \pm \infty \text { if }(H F \pm) \text {, respectively. }
$$

Indeed, it follows from (HF+) that there exist $R>0$ and $C>0$ independent of $n$ such that $f(x, t) t-p F(x, t) \geq 0$ if $|t| \geq R$ and a.e. $x \in \Omega$, and $|f(x, t) t-p F(x, t)| \leq C$ for every $|t| \leq R$ and a.e. $x \in \Omega$. Therefore, since $\left|u_{n}(x)\right| \rightarrow \infty$ a.e. $x \in \Omega_{0}$ and $\left|\Omega_{0}\right|>0$ (note $\left\|v_{0}\right\|_{p}=1$ ), we have (4.2) if $(H F+)$ holds, by applying Fatou's lemma to the following inequality:

$$
(I) \geq \int_{\Omega_{0}} \frac{f\left(x, u_{n}\right) u_{n}-p F\left(x, u_{n}\right)}{\left|u_{n}\right|^{1+q}}\left|v_{n}\right|^{1+q} d x-\frac{C\left|\Omega \backslash \Omega_{0}\right|}{\left\|u_{n}\right\|_{p}^{1+q}} .
$$


In the case of $(H F-)$, by considering $-f$ instead of $f$ as in the above argument, we can show our claim (4.2).

Furthermore, by Hölder's inequality, we have

$$
\begin{aligned}
(I I) & :=\int_{\Omega} \frac{p \tilde{G}\left(x, \nabla u_{n}\right)-\tilde{a}\left(x,\left|\nabla u_{n}\right|\right)\left|\nabla u_{n}\right|^{2}}{\left\|u_{n}\right\|_{p}^{1+q}} d x \\
& \leq H_{0} \int_{\Omega}\left(\left|\nabla v_{n}\right|^{1+q}+\frac{1}{\left\|u_{n}\right\|_{p}^{1+q}}\right) d x \leq H_{0}\left\|\nabla v_{n}\right\|_{p}^{1+q}|\Omega|^{(p-1-q) / p}+o(1) \\
& \leq H_{0}\left\|\nabla v_{0}\right\|_{p}^{1+q}|\Omega|^{(p-1-q) / p}+o(1)
\end{aligned}
$$

in the case of $(H F-)$ because $v_{n} \rightarrow v_{0}$ in $W^{1, p}(\Omega)$, where $q \in[0, p-1]$ and $H_{0}>0$ are constants as in $(H F-)$. Similarly, we obtain

$$
(I I) \geq-H_{0}\left\|\nabla v_{0}\right\|_{p}^{1+q}|\Omega|^{(p-1-q) / p}+o(1)
$$

in the case of $(H F+)$.

Hence, we have a contradiction because of (4.2), (4.4), or (4.5) by taking a limit inferior or superior in the following equality:

$$
o(1)=\frac{p I_{\lambda, m}\left(u_{n}\right)-\left\langle I_{\lambda, m}^{\prime}\left(u_{n}\right), u_{n}\right\rangle}{\left\|u_{n}\right\|_{p}^{1+q}}=(I I)+(I)+(1-p) \int_{\Omega} \frac{h v_{n}}{\left\|u_{n}\right\|_{p}^{q}} d x,
$$

where we use the fact that $\left\|u_{n}\right\| /\left\|u_{n}\right\|_{p}^{1+q}=\left\|v_{n}\right\| /\left\|u_{n}\right\|_{p}^{q}$ is bounded because of $q \geq 0$.

Assume $\lambda \neq 0$ and $(H+)$ or $(H-)$ : because $v_{0}$ is a nontrivial solution of (4.1) with $\lambda \neq 0$, $v_{0}$ is not a constant function, that is, $\left\|\nabla v_{0}\right\|_{p}>0$. Therefore, we have $\left|\nabla u_{n}(x)\right| \rightarrow \infty$ as $n \rightarrow \infty$ for a.e. $x \in \widetilde{\Omega}_{0}:=\left\{x^{\prime} \in \Omega ;\left|\nabla v_{0}\left(x^{\prime}\right)\right| \neq 0\right\}$. Because of $\left|\widetilde{\Omega}_{0}\right|>0$, we can show

$$
\int_{\Omega} \frac{p \tilde{G}\left(x, \nabla u_{n}\right)-\tilde{a}\left(x,\left|\nabla u_{n}\right|\right)\left|\nabla u_{n}\right|^{2}}{\left\|u_{n}\right\|_{p}^{1+q}} d x \longrightarrow \pm \infty \quad \text { if }(H \pm), \text { respectively }
$$

by a similar argument to one for $f$ in the above. In addition, we can easily obtain the following inequality:

$$
\pm \int_{\Omega} \frac{f\left(x, u_{n}\right) u_{n}-p F\left(x, u_{n}\right)}{\left\|u_{n}\right\|_{p}^{1+q}} d x \geq-H_{0}\left\|v_{n}\right\|_{1+q}^{1+q}+o(1)=-H_{0}\left\|v_{0}\right\|_{1+q}^{1+q}+o(1)
$$

in the case of $(H \pm)$, respectively. Hence, we have a contradiction by considering $o(1)=$ $\left(p I_{\lambda, m}\left(u_{n}\right)-\left\langle I_{\lambda, m}^{\prime}\left(u_{n}\right), u_{n}\right\rangle\right) /\left\|u_{n}\right\|_{p}^{1+q}$. 
By a similar way to the case $\int_{\Omega} m d x=0$, we introduce the following approximate functionals on $W^{1, p}(\Omega)$ :

$$
I_{\lambda, m, n}^{ \pm}(u):=I_{\lambda, m}(u) \pm \frac{1}{p n}\|u\|_{p}^{p} \quad \text { for } u \in W^{1, p}(\Omega)
$$

Note $I_{\lambda, m, n}^{ \pm}(u)=I_{\lambda, m \mp 1 /(\lambda n)}(u)$ on $W^{1, p}(\Omega)$ provided $\lambda \neq 0$.

Proposition 4.2. If either $\lambda \neq 0$ and $(H+)$ or $(H F+)$ (resp., either $\lambda \neq 0$ and (H-) or (HF-)) and $\left\{u_{n}\right\}$ satisfies

$$
\begin{gathered}
\sup _{n \in \mathbb{N}} I_{\lambda, m, n}^{+}\left(u_{n}\right)<+\infty, \quad \lim _{n \rightarrow \infty}\left\|\left(I_{\lambda, m, n}^{+}\right)^{\prime}\left(u_{n}\right)\right\|_{W^{1, p}(\Omega)^{*}}=0, \\
\left(\text { resp. } \inf _{n \in \mathbb{N}} I_{\lambda, m, n}^{-}\left(u_{n}\right)>-\infty, \lim _{n \rightarrow \infty}\left\|\left(I_{\lambda, m, n}^{-}\right)^{\prime}\left(u_{n}\right)\right\|_{W^{1, p}(\Omega)^{*}}=0\right),
\end{gathered}
$$

then $\left\{u_{n}\right\}$ is bounded in $W^{1, p}(\Omega)$.

Proof. First, we note that the boundedness of $\left\|u_{n}\right\|_{p}$ guarantees that $\left\|u_{n}\right\|$ is bounded by $\lim _{n \rightarrow \infty}\left\|\left(I_{\lambda, m, n}^{ \pm}\right)^{\prime}\left(u_{n}\right)\right\|_{W^{1, p}(\Omega)^{*}}=0$ (refer to (3.6) as in the proof of Proposition 3.3). Moreover, because of the following equality:

$$
\begin{aligned}
& \frac{p I_{\lambda, m, n}^{ \pm}\left(u_{n}\right)-\left\langle\left(I_{\lambda, m, n}^{ \pm}\right)^{\prime}\left(u_{n}\right), u_{n}\right\rangle}{\left\|u_{n}\right\|_{p}^{1+q}}=(1-p) \int_{\Omega} \frac{h v_{n}}{\left\|u_{n}\right\|_{p}^{q}} d x \\
& \quad+\int_{\Omega} \frac{p \tilde{G}\left(x, \nabla u_{n}\right)-\tilde{a}\left(x,\left|\nabla u_{n}\right|\right)\left|\nabla u_{n}\right|^{2}}{\left\|u_{n}\right\|_{p}^{1+q}} d x+\int_{\Omega} \frac{f\left(x, u_{n}\right) u_{n}-p F\left(x, u_{n}\right)}{\left\|u_{n}\right\|_{p}^{1+q}} d x
\end{aligned}
$$

we can prove the boundedness of $\left\|u_{n}\right\|_{p}$ by the same argument as in Proposition 4.1.

Proof of Theorem 1.2. Because of $\lambda m=(-\lambda)(-m)$, we may assume $\lambda \geq 0$. In the case where $\int_{\Omega} m d x \neq 0$ and $\mu_{k}(m)<\lambda<\mu_{k+1}(m)$ for some $k \in \mathbb{N}$, the proof of Theorem 1.1 implies the existence of a critical point of $I_{\lambda, m}$ because $I_{\lambda, m}$ satisfies the Palais-Smale condition by Proposition 4.1. Concerning other cases, in the next section, we will prove the existence of a bounded sequence $\left\{u_{n}\right\}$ satisfying $\left(I_{\lambda, m, n}^{+}\right)^{\prime}\left(u_{n}\right) \rightarrow 0$ or $\left(I_{\lambda, m, n}^{-}\right)^{\prime}\left(u_{n}\right) \rightarrow 0$ in $W^{1, p}(\Omega)^{*}$ as $n \rightarrow$ $\infty$. Because $I_{\lambda, m}$ is bounded on a bounded set, we may assume that $I_{\lambda, m}\left(u_{n}\right)$ converges to some $c \in \mathbb{R}$ by choosing a subsequence. In addition, by noting the inequality $\left\|I_{\lambda, m}^{\prime}\left(u_{n}\right)\right\|_{W^{1, p}(\Omega)^{*}} \leq$ $\left\|\left(I_{\lambda, m, n}^{ \pm}\right)^{\prime}\left(u_{n}\right)\right\|_{W^{1, p}(\Omega)^{*}}+\left\|u_{n}\right\|_{p}^{p-1} / n$, we easily see that $\left\{u_{n}\right\}$ is a bounded Palais-Smale sequence of $I_{\lambda, m}$. Therefore, $I_{\lambda, m}$ has a critical point since $I_{\lambda, m}$ satisfies the Palais-Smale condition by Proposition 4.1. 


\section{Construction of a Bounded Palais-Smale Sequence}

In this section, due to the reason stated in the proof of Theorem 1.2, we will construct a bounded sequence $\left\{u_{n}\right\}$ satisfying $\left(I_{\lambda, m, n}^{+}\right)^{\prime}\left(u_{n}\right) \rightarrow 0$ or $\left(I_{\lambda, m, n}^{-}\right)^{\prime}\left(u_{n}\right) \rightarrow 0$ in $W^{1, p}(\Omega)^{*}$ as $n \rightarrow \infty$. It implies the existence of a bounded Palais-Smale sequence of $I_{\lambda, m}$.

\subsection{The Case $\lambda=0$}

\section{Assume (HF+)}

In this $\mathrm{c}$ ase, we can show that for each $n \in \mathbb{N}, I_{\lambda, m, n}^{+}$has a global minimizer $u_{n}$. Indeed, for $0<\varepsilon<1 /(p n)$, there exists $C_{\varepsilon}>0$ such that $I_{\lambda, m, n}^{+}(u) \geq C_{0}\|\nabla u\|_{p}^{p} /(p(p-1))+(1 /(p n)-$ $\varepsilon)\|u\|_{p}^{p}-\|h\|_{\infty}\|u\|_{1}-C_{\varepsilon}$ for every $u \in W^{1, p}(\Omega)$ by (1.1), (1.16) and $\lambda=0$ (refer to the inequality as in the proof of Lemma 3.5). This means that $I_{\lambda, m, n}^{+}$is coercive and bounded from below on $W^{1, p}(\Omega)$. Therefore, $I_{\lambda, m, n}^{+}$has a global minimizer $u_{n}$ since $I_{\lambda, m, n}^{+}$is w.l.s.c. on $W^{1, p}(\Omega)$ as the same reason in Lemma 3.6.

Furthermore, because of $\left(I_{\lambda, m, n}^{+}\right)^{\prime}\left(u_{n}\right)=0$ in $W^{1, p}(\Omega)^{*}$ and $I_{\lambda, m, n}^{+}\left(u_{n}\right)=\min _{W^{1, p}(\Omega)}$ $I_{\lambda, m, n}^{+} \leq I_{\lambda, m, n}^{+}(0)=0$, it follows from Proposition 4.2 that $\left\{u_{n}\right\}$ is bounded.

\section{Assume (HF-)}

Choose $n_{0} \in \mathbb{N}$ such that $1 / n_{0}<c(1)=\mu_{2}(1)$, where $c(1)$ is the second eigenvalue of $(E V ; 1)$ (so the weight function $m \equiv 1$ and see (2.22) for the definition). Then, by noting that $I_{0, m, n_{0}}^{-}=$ $I_{1 / n_{0}, 1}$, we have

$$
\alpha:=\inf \left\{I_{0, m, n_{0}}^{-}(u) ; u \in Y(c(1), 1)\right\}>-\infty
$$

by Lemma 3.8, where $Y(c(1), 1)$ is a subset defined by (3.14) with the weight $m \equiv 1$, that is,

$$
Y(c(1), 1):=\left\{u \in W^{1, p}(\Omega) ; \int_{\Omega} a_{\infty}|\nabla u|^{p} d x \geq c(1)\|u\|_{p}^{p}\right\} .
$$

Moreover, $\inf \left\{I_{0, m, n}^{-}(u) ; u \in Y(c(1), 1)\right\} \geq \alpha$ for every $n \geq n_{0}$ holds because $I_{0, m, n}^{-}(u) \geq I_{0, m, n_{0}}^{-}(u)$ for every $u \in W^{1, p}(\Omega)$. Since $\int_{\Omega} F(x, u) d x=o(1)\|u\|_{p}^{p}$ as $\|u\|_{p} \rightarrow \infty$ by (1.1), there exists $T_{n}>0$ such that $I_{0, m, n}^{-}\left( \pm T_{n}\right)=-T_{n}^{p}(|\Omega| /(n p)-o(1))<\alpha-2$.

Define

$$
\begin{gathered}
\Sigma_{n}:=\left\{g \in C\left([0,1], W^{1, p}(\Omega)\right) ; g(0)=T_{n}, g(1)=-T_{n}\right\}, \\
c_{n}:=\inf _{g \in \Sigma_{n}} \max _{t \in[0,1]} I_{0, m, n}^{-}(g(t))
\end{gathered}
$$

for $n \geq n_{0}$. By the definition of $c(1)$, we easily see that $g([0,1]) \cap Y(c(1), 1) \neq \emptyset$ for every $g \in \Sigma_{n}$ (refer to [6] or Lemma 3.4). Hence,

$$
c_{n} \geq \inf \left\{I_{0, m, n}^{-}(u) ; u \in Y(c(1), 1)\right\} \geq \alpha>I_{0, m, n}\left( \pm T_{n}\right)
$$


holds, whence $c_{n}$ is bounded from below. Moreover, by applying Ekeland's variational principle to each $I_{0, m, n}^{-}$, we can obtain a sequence $\left\{u_{n}\right\}$ satisfying $\left|I_{0, m, n}^{-}\left(u_{n}\right)-c_{n}\right|<1 / n$ and $\left\|\left(I_{0, m, n}^{-}\right)^{\prime}\left(u_{n}\right)\right\|_{W^{1, p}(\Omega)^{*}}<1 / n$. Since $c_{n}$ is bounded from below, it follows from Proposition 4.2 that $\left\{u_{n}\right\}$ is bounded. As a result, we can construct a bounded sequence $\left\{u_{n}\right\}$ satisfying $\left(I_{0, m, n}^{-}\right)^{\prime}\left(u_{n}\right) \rightarrow 0$ as $n \rightarrow \infty$ in $W^{1, p}(\Omega)^{*}$.

5.2. The Case $\lambda=\lambda^{*}(m)=\mu_{1}(m)$ with $\int_{\Omega} m d x<0$

Assume $(\mathrm{H}+)$ or $(\mathrm{HF}+)$

Since we see that $I_{\lambda, m, n}^{+}=I_{\lambda, m-1 /(n \lambda)}$ and $\lambda^{*}(m-1 /(n \lambda))>\lambda^{*}(m)=\lambda>0$ (according to Lemma 2.5), $I_{\lambda, m, n}^{+}$is coercive, bounded from below and w.l.s.c. on $W^{1, p}(\Omega)$ by Lemma 3.6. Thus, we obtain a global minimizer $u_{n}$ of $I_{\lambda, m, n}^{+}$for sufficiently large $n$ such that $\mid\{m-1 /(n \lambda)>$ $0\} \mid>0$. Because of $I_{\lambda, m, n}^{+}\left(u_{n}\right) \leq I_{\lambda, m, n}^{+}(0)=0$ for every $n$, Proposition 4.2 guarantees that $\left\{u_{n}\right\}$ is bounded.

Assume (H-) or (HF-)

First, we note that $I_{\lambda, m, n}^{-}=I_{\lambda, m+1 /(n \lambda)}$ and $0<\lambda^{*}(m+1 /(n \lambda))<\lambda^{*}(m)=\lambda$ by Lemma 2.5 for sufficiently large $n$ such that $\int_{\Omega}(m+1 /(n \lambda)) d x<0$. Moreover, it follows from Proposition 2.10 and $\mu_{1}(m)<\mu_{2}(m)$ that there exists an $n_{0} \in \mathbb{N}$ satisfying $\int_{\Omega} m+1 /\left(n_{0} \lambda\right) d x<0$ and

$$
\lambda^{*}\left(m+\frac{1}{n \lambda}\right)<\lambda=\mu_{1}(m)<\mu_{2}\left(m+\frac{1}{n_{0} \lambda}\right) \leq \mu_{2}\left(m+\frac{1}{n \lambda}\right) \leq \mu_{2}(m)
$$

for every $n \geq n_{0}$. By applying Theorem 1.1 to each case of a weight $m+1 /(n \lambda)$ (note that $\lambda$ is not an eigenvalue of $(E V ; m+1 /(n \lambda))$ by $(5.5)$, there exists $u_{n}$ satisfying $\left(I_{\lambda, m, n}^{-}\right)^{\prime}\left(u_{n}\right)=0$ (note $I_{\lambda, m, n}^{-}=I_{\lambda, m+1 /(n \lambda)}$ ) and

$$
I_{\lambda, m, n}^{-}\left(u_{n}\right)=c_{n} \geq \inf \left\{I_{\lambda, m, n}^{-}(u) ; u \in Y\left(\mu_{2}\left(m_{n_{0}}\right), m_{n_{0}}\right)\right\}
$$

where the last inequality follows from Lemma 3.4 with $m_{n_{0}}:=m+1 /\left(n_{0} \lambda\right)$. On the other hand, because $I_{\lambda, m, n}^{-}(u) \geq I_{\lambda, m, n_{0}}^{-}(u)=I_{\lambda, m_{n_{0}}}(u)$ for every $u \in W^{1, p}(\Omega)$ and $n \geq n_{0}$, we have

$$
c_{n} \geq \inf \left\{I_{\lambda, m_{n_{0}}}(u) ; u \in Y\left(\mu_{2}\left(m_{n_{0}}\right), m_{n_{0}}\right)\right\}>-\infty
$$

for every $n \geq n_{0}$, where the last inequality follows from Lemma 3.8. Thus, $c_{n}$ is bounded from below. Hence, Proposition 4.2 guarantees the boundedness of $\left\{u_{n}\right\}$. 
5.3. The Case $\lambda=\mu_{k+1}(m)$ with $\int_{\Omega} m d x \neq 0$

Assume $(\mathrm{H}+)$ or $(\mathrm{HF}+)$

We may assume $\mu_{k}(m)<\mu_{k+1}(m)=\lambda$ by taking $k$ anew if necessary (note that we have already proved the case of $\mu_{k}(m)<\lambda<\mu_{k+1}(m)$ in Section 4). Here, we can choose an $n_{0} \in \mathbb{N}$ such that $\int_{\Omega}(m-1 /(n \lambda)) d x \neq 0,|\{m-1 /(n \lambda)>0\}|>0$ and

$$
\mu_{k}\left(m-\frac{1}{n \lambda}\right) \leq \mu_{k}\left(m-\frac{1}{n_{0} \lambda}\right)<\lambda-\frac{1}{n\|m\|_{\infty}}<\lambda=\mu_{k+1}(m) \leq \mu_{k+1}\left(m-\frac{1}{n \lambda}\right)
$$

for every $n \geq n_{0}$ by $\int_{\Omega} m d x \neq 0$ and Proposition 2.10 (i), (iii). Note the following inequality:

$$
I_{\lambda, m, n_{0}}^{+}(u) \geq I_{\lambda, m, n}^{+}(u) \geq I_{\lambda-1 /\left(n\|m\|_{\infty}\right), m}(u)
$$

for every $u \in W^{1, p}(\Omega)$ and $n \geq n_{0}$, where the last inequality is obtained by $\|u\|_{p}^{p} \geq \int_{\Omega} m|u|^{p} d x /$ $\|m\|_{\infty}$. Let $n \geq n_{0}$. It follows from Lemma 3.8 and (5.8) that $I_{\lambda-1 /\left(n\|m\|_{\infty}\right), m}$ is bounded from below on $Y(\lambda, m)$. Hence, (5.9) yields that $I_{\lambda, m, n}^{+}$is also bounded from below on $Y(\lambda, m)$, namely,

$$
\alpha_{n}:=\inf \left\{I_{\lambda, m, n}^{+}(u) ; u \in Y(\lambda, m)\right\}>-\infty
$$

On the other hand, because of $\mu_{k}\left(m-1 /\left(n_{0} \lambda\right)\right)<\lambda$ (see (5.8)), Lemma 3.5 guarantees the existence of $g_{0} \in \mathcal{F}_{k}\left(m-1 /\left(n_{0} \lambda\right)\right)$ satisfying

$$
\max _{z \in S^{k-1}} I_{\lambda, m, n_{0}}^{+}\left(T g_{0}(z)\right)=\max _{z \in S^{k-1}} I_{\lambda, m-1 /\left(n_{0} \lambda\right)}\left(T g_{0}(z)\right) \longrightarrow-\infty \quad \text { as }|T| \longrightarrow \infty
$$

Thus, for each $n \geq n_{0}$, we can take $T_{n}>0$ such that

$$
\max _{z \in S^{k-1}} I_{\lambda, m, n}^{+}\left(T_{n} g_{0}(z)\right) \leq \max _{z \in S^{k-1}} I_{\lambda, m, n_{0}}^{+}\left(T_{n} g_{0}(z)\right) \leq \alpha_{n}-1
$$

(note (5.9) for the first inequality). Set

$$
\begin{gathered}
\Sigma_{n}:=\left\{g \in C\left(S_{+}^{k}, W^{1, p}(\Omega)\right) ;\left.g\right|_{S^{k-1}}=T_{n} g_{0}\right\}, \\
c_{n}^{+}:=\inf _{g \in \Sigma_{n}} \max _{z \in S_{+}^{k}} I_{\lambda, m, n}^{+}(g(z))
\end{gathered}
$$

for $n \geq n_{0}$. Since $g\left(S_{+}^{k}\right) \cap \Upsilon(\lambda, m) \neq \emptyset$ for every $g \in \Sigma_{n}$ by Lemma 3.4 and $\lambda=\mu_{k+1}(m)$, we have $c_{n}^{+} \geq \alpha_{n}>\max _{z \in S^{k-1}} I_{\lambda, m, n}^{+}\left(T_{n} g_{0}(z)\right)$. Therefore, Ekeland's variational principle (refer to [25, Theorem 4.3]) guarantees the existence of $u_{n}$ satisfying $\left|I_{\lambda, m, n}^{+}\left(u_{n}\right)-c_{n}\right|<1 / n$ and $\left\|\left(I_{\lambda, m, n}^{+}\right)^{\prime}\left(u_{n}\right)\right\|_{W^{1, p}(\Omega)^{*}}<1 / n$. 
Finally, to show the boundedness of $\left\{u_{n}\right\}$ due to Proposition 4.2, we will prove that $c_{n}^{+}$ is bounded from above. For each $n \geq n_{0}$, we define a continuous map $g_{n}$ from $S_{+}^{k}$ to $W^{1, p}(\Omega)$ by

$$
g_{n}(z):= \begin{cases}\left(1-z_{k+1}\right) T_{n} g_{0}\left(\frac{z^{\prime}}{\sqrt{1-z_{k+1}^{2}}}\right) & \text { for } z=\left(z^{\prime}, z_{k+1}\right) \in S_{+}^{k} \text { with } 0 \leq z_{k+1}<1 \\ 0 & \text { for } z=\left(z^{\prime}, z_{k+1}\right) \in S_{+}^{k} \text { with } z_{k+1}=1 .\end{cases}
$$

Then, $g_{n} \in \Sigma_{n}$ holds. This leads to

$$
c_{n}^{+} \leq \sup _{t \geq 0, z \in S^{k-1}} I_{\lambda, m, n}^{+}\left(\operatorname{tg} g_{0}(z)\right) \leq \sup _{t \geq 0, z \in S^{k-1}} I_{\lambda, m, n_{0}}^{+}\left(\operatorname{tg} g_{0}(z)\right)<+\infty
$$

because of (5.9), (5.11), and the compactness of $g_{0}\left(S^{k-1}\right)$.

Assume (H-) or (HF-)

Because the case of $\mu_{1}(m)=\lambda^{*}(m)$ is already shown (see Sections 5.1 and 5.2), We may assume $(0<) \mu_{k}(m)=\lambda<\mu_{k+1}(m)$ for some $k \geq 2$ by taking $k$ anew if necessary. Here, we can choose an $n_{0} \in \mathbb{N}$ such that $\int_{\Omega}(m+1 /(n \lambda)) d x \neq 0$ and

$$
\mu_{k}\left(m+\frac{1}{n \lambda}\right) \leq \mu_{k}(m)=\lambda<\mu_{k+1}\left(m+\frac{1}{n_{0} \lambda}\right) \leq \mu_{k+1}\left(m+\frac{1}{n \lambda}\right) \leq \mu_{k+1}(m)
$$

for every $n \geq n_{0}$ by $\int_{\Omega} m d x \neq 0$ and Proposition 2.10 (i), (iii). Moreover, we note the following inequality:

$$
I_{\lambda, m, n_{0}}^{-}(u) \leq I_{\lambda, m, n}^{-}(u)=I_{\lambda, m+1 /(n \curlywedge)}(u) \leq I_{\lambda+1 /\left(n\|m\|_{\infty}\right), m}(u)
$$

for every $u \in W^{1, p}(\Omega)$ and $n \geq n_{0}$. It follows from Lemma 3.8 and (5.16) (note (5.17) also) that $I_{\lambda, m, n_{0}}^{-}=I_{\lambda, m_{0}}$ is bounded from below on $Y\left(\mu_{k+1}\left(m_{0}\right), m_{0}\right)$ with $m_{0}:=m+1 /\left(n_{0} \lambda\right)$. Hence, (5.17) implies

$$
\begin{aligned}
& \inf \left\{I_{\lambda, m, n}^{-}(u) ; u \in Y\left(\mu_{k+1}\left(m_{0}\right), m_{0}\right)\right\} \\
& \quad \geq \inf \left\{I_{\lambda, m, n_{0}}^{-}(u) ; u \in Y\left(\mu_{k+1}\left(m_{0}\right), m_{0}\right)\right\}=: \alpha_{0}>-\infty
\end{aligned}
$$

for every $n \geq n_{0}$. Because of $\lambda+1 /\left(n\|m\|_{\infty}\right)>\lambda=\mu_{k}(m)$, there exist $g_{n} \in \mathcal{F}_{k}(m)$ and $T_{n}>0$ such that

$$
\max _{z \in S^{k-1}} I_{\lambda, m, n}^{-}\left(T_{n} g_{n}(z)\right) \leq \max _{z \in S^{k-1}} I_{\lambda+1 /\left(n\|m\|_{\infty}\right), m}\left(T_{n} g_{n}(z)\right)<\alpha_{0}-1
$$


by Lemma 3.5. Define

$$
\begin{gathered}
\Sigma_{n}:=\left\{g \in C\left(S_{+}^{k}, W^{1, p}(\Omega)\right) ;\left.g\right|_{S^{k-1}}=T_{n} g_{n}\right\}, \\
c_{n}^{-}:=\inf _{g \in \Sigma_{n}} \max _{z \in S_{+}^{k}} I_{\lambda, m, n}^{-}(g(z))
\end{gathered}
$$

for $n \geq n_{0}$. Then, $c_{n}^{-} \geq \alpha_{0}$ occurs (see (5.18)) since $g\left(S_{+}^{k}\right) \cap Y\left(\mu_{k+1}\left(m_{0}\right), m_{0}\right) \neq \emptyset$ for every $g \in \Sigma_{n}$ by Lemma 3.4. This means that $c_{n}^{-}$is bounded from below. Consequently, we can obtain a desired bounded sequence by the same argument as in Sections 5.1 and 5.2.

\subsection{The Case (iii) as in Theorem 1.2}

First, note that we are assuming the hypothesis $(H+)$ or $(H F+)$ in this case (iii). In addition, as the reason in the proof of Theorem 1.2, it suffices to handle with $\lambda>0$.

Let $k \in \mathbb{N}$ satisfy $\mu_{k}(m)<\lambda \leq \mu_{k+1}(m)$. According to Proposition 2.10 (i) and (ii), we can take an $n_{0} \in \mathbb{N}$ such that $|\{m-1 /(n \lambda)>0\}|>0$ and

$$
\mu_{k}\left(m-\frac{1}{2 n \lambda}\right) \leq \mu_{k}\left(m-\frac{1}{n_{0} \lambda}\right)<\lambda-\frac{1}{2 n\|m\|_{\infty}}<\lambda \leq \mu_{k+1}(m) \leq \mu_{k+1}\left(m-\frac{1}{2 n \lambda}\right)
$$

for every $n \geq n_{0}$. The following inequality follows from the easy estimates:

$$
I_{\lambda, m, n_{0}}^{+}(u) \geq I_{\lambda, m, n}^{+}(u)=I_{\lambda, m-1 /(n \lambda)}(u) \geq I_{\lambda-1 /\left(2 n\|m\|_{\infty}\right), m-1 /(2 n \lambda)}(u)
$$

for every $u \in W^{1, p}(\Omega)$ and $n \geq n_{0}$. Let $n \geq n_{0}$ and set $m_{n}:=m-1 /(2 n \lambda)$. Because of (5.21), Lemma 3.8 implies that $I_{\lambda-1 /\left(2 n\|m\|_{\infty}\right), m_{n}}$ is bounded from below on $Y\left(\mu_{k+1}\left(m_{n}\right), m_{n}\right)$ with (note $\left.\int_{\Omega} m_{n} d x \neq 0\right)$. Hence, (5.22) yields that

$$
\alpha_{n}:=\inf \left\{I_{\lambda, m, n}^{+}(u) ; u \in Y\left(\mu_{k+1}\left(m_{n}\right), m_{n}\right)\right\}>-\infty
$$

for each $n \geq n_{0}$. On the other hand, because of $\mu_{k}\left(m-1 /\left(n_{0} \lambda\right)\right)<\lambda$ (see (5.21)), Lemma 3.5 guarantees the existence of $g_{0} \in \mathcal{F}_{k}\left(m-1 /\left(n_{0} \lambda\right)\right)$ satisfying

$$
\max _{z \in S^{k-1}} I_{\lambda, m, n_{0}}^{+}\left(T g_{0}(z)\right)=\max _{z \in S^{k-1}} I_{\lambda, m-1 /\left(n_{0} \lambda\right)}\left(T g_{0}(z)\right) \longrightarrow-\infty \quad \text { as } T \longrightarrow \infty
$$

Therefore, for each $n \geq n_{0}$, we can choose $T_{n}>0$ such that

$$
\max _{z \in S^{k-1}} I_{\lambda, m, n}^{+}\left(T_{n} g_{0}(z)\right) \leq \max _{z \in S^{k-1}} I_{\lambda, m, n_{0}}^{+}\left(T_{n} g_{0}(z)\right) \leq \alpha_{n}-1,
$$


(note (5.22) for the first inequality). Set

$$
\begin{gathered}
\Sigma_{n}:=\left\{g \in C\left(S_{+}^{k}, W^{1, p}(\Omega)\right) ;\left.g\right|_{S^{k-1}}=T_{n} g_{0}\right\}, \\
c_{n}^{+}:=\inf _{g \in \Sigma_{n}} \max _{z \in S_{+}^{k}} I_{\lambda, m, n}^{+}(g(z))
\end{gathered}
$$

for $n \geq n_{0}$. Since $g\left(S_{+}^{k}\right) \cap Y\left(\mu_{k+1}\left(m_{n}\right), m_{n}\right) \neq \emptyset$ for every $g \in \Sigma_{n}$ by Lemma 3.4, we have $c_{n}^{+} \geq$ $\alpha_{n}>\max _{z \in S^{k-1}} I_{\lambda, m, n}^{+}\left(T_{n} g_{0}(z)\right)$. Moreover, by the same argument as in Section 5.3 (note (5.24)), we have

$$
c_{n}^{+} \leq \sup _{t \geq 0, z \in S^{k-1}} I_{\lambda, m, n}^{+}\left(\operatorname{tg} g_{0}(z)\right) \leq \sup _{t \geq 0, z \in S^{k-1}} I_{\lambda, m, n_{0}}^{+}\left(\operatorname{tg} g_{0}(z)\right)<+\infty
$$

and hence our conclusion is shown.

Remark 5.1. If $\int_{\Omega} m d x=0$ holds, then we can not show the continuity of $\mu_{k}(m)$ with respect to $m$ (refer to Proposition 2.10). Hence, we are not able to construct a bounded Palais-Smale sequence under $(H-)$ or $(H F-)$. However, if we have the additional information about the existence of a suitable $m^{\prime} \in L^{\infty}(\Omega)$ such that $m^{\prime} \geq m$ in $\Omega, \int_{\Omega} m^{\prime} d x \neq 0$ and $\mu_{k}(m) \leq \lambda<$ $\mu_{k+1}\left(m^{\prime}\right)$ when $\mu_{k}(m) \leq \lambda<\mu_{k+1}(m)$ occurs, then we can still easily prove that equation $(P ; \lambda, m, h)$ has a solution in the case also where $\lambda \neq 0, \int_{\Omega} m d x=0$ and $(H-)$ or $(H F-)$. In fact, let $0<\mu_{k}(m) \leq \lambda<\mu_{k+1}\left(m^{\prime}\right)$ for some $k \geq 2$. Note the following inequality:

$$
I_{\lambda+1 /\left(n\|m\|_{\infty}\right), m}(u) \geq I_{\lambda, m, n}^{-}(u) \geq I_{\lambda, m^{\prime}}(u)-\frac{1}{n p}\|u\|_{p}^{p}=I_{\lambda, m^{\prime}-1 /(n \lambda)}(u)
$$

for every $u \in W^{1, p}(\Omega)$ and $n$. Fix $n_{0} \in \mathbb{N}$ such that $\int_{\Omega} m^{\prime}-1 /\left(n_{0} \lambda\right) d x>0$ and $\mid\left\{m^{\prime}-1 /\left(n_{0} \lambda\right)>\right.$ $0\} \mid>0$. Set $m_{0}^{\prime}:=m^{\prime}-1 /\left(n_{0} \lambda\right)$. Because of $\lambda<\mu_{k+1}\left(m^{\prime}\right) \leq \mu_{k+1}\left(m_{0}^{\prime}\right)$ (the last inequality follows from Proposition 2.10 (i)), Lemma 3.8 implies that $I_{\lambda, m_{0}^{\prime}}$ is bounded from below on $Y\left(\mu_{k+1}\left(m_{0}^{\prime}\right), m_{0}^{\prime}\right)$ (note $\left.\int_{\Omega} m_{0}^{\prime} d x>0\right)$. By combining this fact and (5.28), we have

$$
\begin{aligned}
& \inf _{n \geq n_{0}} \inf \left\{I_{\lambda, m, n}^{-}(u) ; u \in Y\left(\mu_{k+1}\left(m_{0}^{\prime}\right), m_{0}^{\prime}\right)\right\} \\
& \quad \geq \inf \left\{I_{\lambda, m_{0}^{\prime}}(u) ; u \in Y\left(\mu_{k+1}\left(m_{0}^{\prime}\right), m_{0}^{\prime}\right)\right\}>-\infty .
\end{aligned}
$$

Because of $\lambda+1 /\left(n\|m\|_{\infty}\right)>\lambda \geq \mu_{k}(m)$, for each $n \geq n_{0}$, we can take a $g_{n} \in \mathscr{F}_{k}(m)$ satisfying

$$
\max _{z \in S^{k-1}} I_{\lambda, m, n}^{-}\left(T g_{n}(z)\right) \leq \max _{z \in S^{k-1}} I_{\lambda+1 /\left(n\|m\|_{\infty}\right), m}\left(T g_{n}(z)\right) \longrightarrow-\infty
$$

as $T \rightarrow \infty$ by Lemma 3.5.

Since any extension $g \in C\left(S_{+}^{k}, W^{1, p}(\Omega)\right)$ of $T g_{n}(T>0)$ links $Y\left(\mu_{k+1}\left(m_{0}^{\prime}\right), m_{0}^{\prime}\right)$ by Lemma 3.4, we can construct a desired sequence by the same argument as in Section 5.3 under $(H-)$ or $(H F-)$. 


\section{Acknowledgments}

The author would like to express her sincere thanks to Professor Shizuo Miyajima for helpful comments and encouragement. The author thanks the referees for his helpful comments and suggestions.

\section{References}

[1] D. Motreanu and N. S. Papageorgiou, "Multiple solutions for nonlinear Neumann problems driven by a nonhomogeneous differential operator," Proceedings of the American Mathematical Society, vol. 139, no. 10, pp. 3527-3535, 2011.

[2] L. Damascelli, "Comparison theorems for some quasilinear degenerate elliptic operators and applications to symmetry and monotonicity results," Annales de l'Institut Henri Poincaré, vol. 15, no. 4, pp. 493-516, 1998.

[3] D. Motreanu, V. V. Motreanu, and N. S. Papageorgiou, "Papageorgiou, multiple constant sign and nodal solutions for nonlinear neumann eigenvalue problems," Annali della Scuola Normale Superiore di Pisa, vol. 10, no. 5, pp. 729-755, 2011.

[4] S. Miyajima, D. Motreanu, and M. Tanaka, "Multiple existence results of solutions for the Neumann problems via super- and sub-solutions," Journal of Functional Analysis, vol. 262, pp. 1921-1953, 2012.

[5] S. T. Robinson, "On the second eigenvalue for nonhomogeneous quasi-linear operators," SIAM Journal on Mathematical Analysis, vol. 35, no. 5, pp. 1241-1249, 2004.

[6] M. Tanaka, "The antimaximum principle and the existence of a solution for the generalized $p$-Laplace equations with indefinite weight," Differential Equations \& Applications, vol. 4, no. 4, 2012.

[7] T. Godoy, J.-P. Gossez, and S. Paczka, "On the antimaximum principle for the $p$-Laplacian with indefinite weight," Nonlinear Analysis, vol. 51, no. 3, pp. 449-467, 2002.

[8] A. Anane and A. Dakkak, "Nonresonance conditions on the potential for a Neumann problem," in Partial Differential Equations, vol. 229 of Lecture Notes in Pure and Applied Mathematics, pp. 85-102, Dekker, New York, NY, USA, 2002.

[9] J.-P. Gossez and P. Omari, "A necessary and sufficient condition of nonresonance for a semilinear Neumann problem," Proceedings of the American Mathematical Society, vol. 114, no. 2, pp. 433-442, 1992.

[10] P. Omari and F. Zanolin, "Nonresonance conditions on the potential for a second-order periodic boundary value problem," Proceedings of the American Mathematical Society, vol. 117, no. 1, pp. 125135, 1993.

[11] A. Ambrosetti and G. Prodi, A Primer of Nonlinear Analysis, vol. 34 of Cambridge Studies in Advanced Mathematics, Cambridge University Press, Cambridge, UK, 1995.

[12] Y. Chen and M. Wang, "Large solutions for quasilinear elliptic equation with nonlinear gradient term," Nonlinear Analysis, vol. 12, no. 1, pp. 455-463, 2011.

[13] P. Y. H. Pang, Y. Wang, and J. Yin, "Periodic solutions for a class of reaction-diffusion equations with P-Laplacian," Nonlinear Analysis, vol. 11, no. 1, pp. 323-331, 2010.

[14] G. Jia, Q. Zhao, and C.-Y. Dai, "Singular quasilinear elliptic problems with indefinite weights and critical potential," Acta Mathematicae Applicatae Sinica, vol. 28, pp. 157-164, 2012.

[15] G. Zhang, S. Man, and W. Zhang, "On a class of critical singular quasilinear elliptic problem with indefinite weights," Nonlinear Analysis, vol. 74, no. 14, pp. 4771-4784, 2011.

[16] P. Drábek and S. B. Robinson, "Resonance problems for the $p$-Laplacian," Journal of Functional Analysis, vol. 169, no. 1, pp. 189-200, 1999.

[17] M. Struwe, Variational Methods, Springer, New York, NY, USA, 1999.

[18] S. E. Habib and N. Tsouli, "On the spectrum of the $p$-Laplacian operator for Neumann eigenvalue problems with weights," Electronic Journal of Differential Equations, vol. 2005, pp. 181-190, 2005.

[19] M. Arias, J. Campos, M. Cuesta, and J.-P. Gossez, "An asymmetric Neumann problem with weights," Annales de l'Institut Henri Poincaré, vol. 25, no. 2, pp. 267-280, 2008.

[20] J.-N. Corvellec, "A general approach to the min-max principle," Zeitschrift für Analysis und ihre Anwendungen, vol. 16, no. 2, pp. 405-433, 1997.

[21] K. Perera, R. P. Agarwal, and D. O’Regan, Morse Theoretic Aspects of p-Laplacian Type Operators, vol. 161 of Mathematical Surveys and Monographs, American Mathematical Society, Providence, RI, USA, 2010. 
[22] A. Szulkin, "Ljusternik-Schnirelmann theory on $C^{1}$-manifolds," Annales de l'Institut Henri Poincaré, vol. 5, no. 2, pp. 119-139, 1988.

[23] G. M. Lieberman, "Boundary regularity for solutions of degenerate elliptic equations," Nonlinear Analysis, vol. 12, no. 11, pp. 1203-1219, 1988.

[24] E. Casas and L. A. Fernandez, "A Green's formula for quasilinear elliptic operators," Journal of Mathematical Analysis and Applications, vol. 142, no. 1, pp. 62-73, 1989.

[25] J. Mawhin and M. Willem, Critical Point Theory and Hamiltonian Systems, Springer, New York, NY, USA, 1989. 


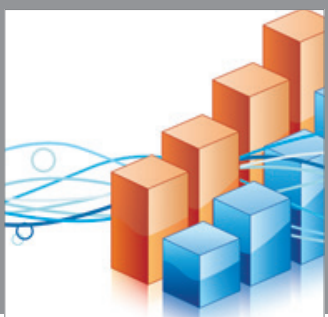

Advances in

Operations Research

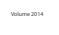

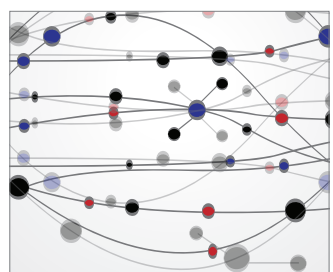

\section{The Scientific} World Journal
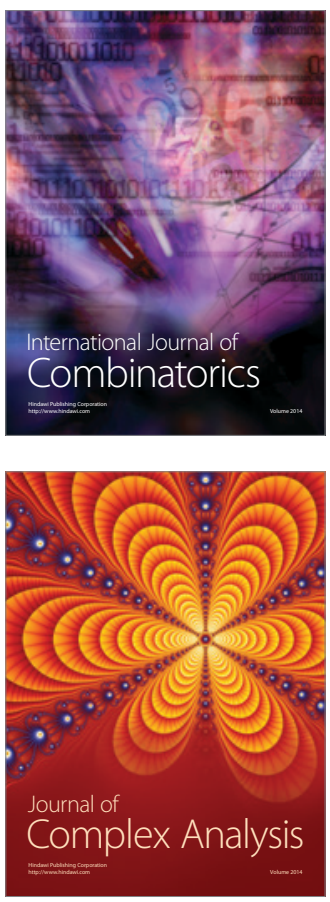

International Journal of

Mathematics and

Mathematical

Sciences
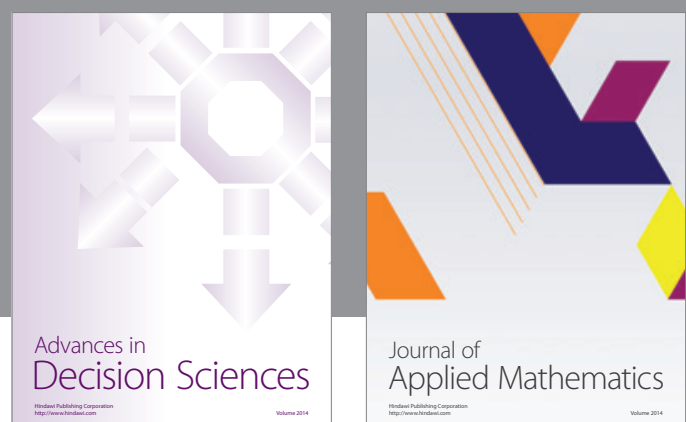

Journal of

Applied Mathematics
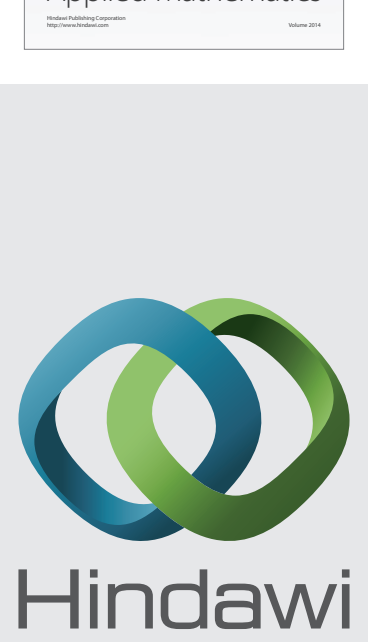

Submit your manuscripts at http://www.hindawi.com
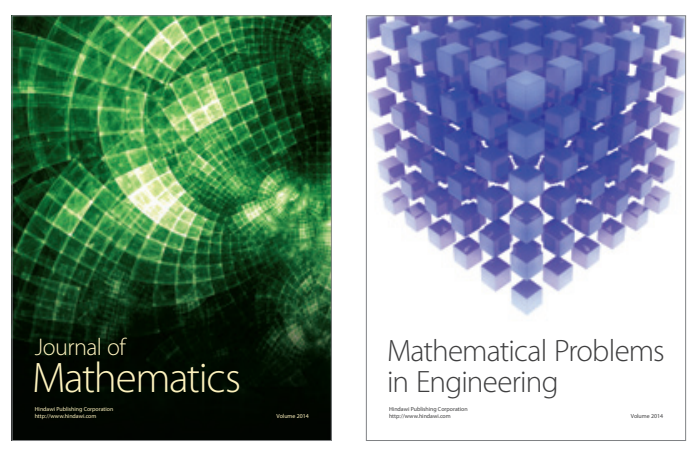

Mathematical Problems in Engineering
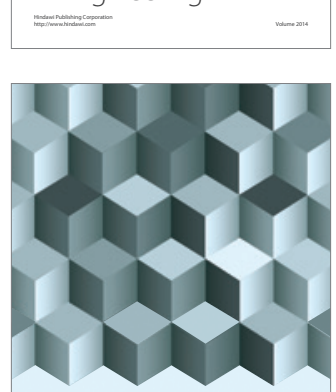

Journal of

Function Spaces
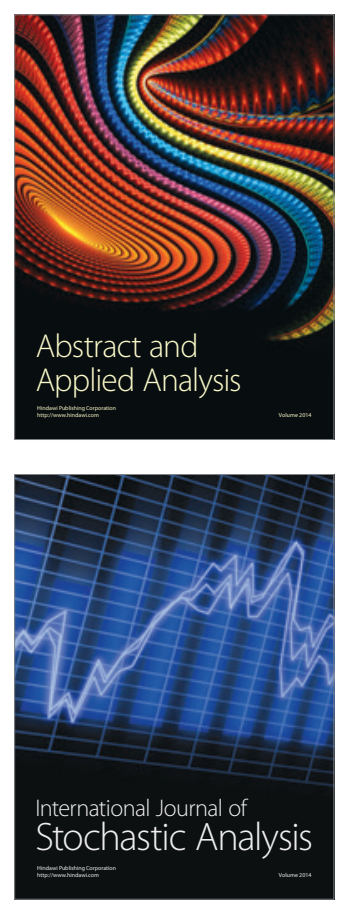

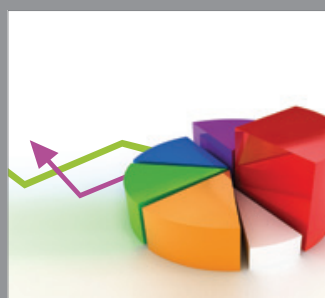

ournal of

Probability and Statistics

Promensencen
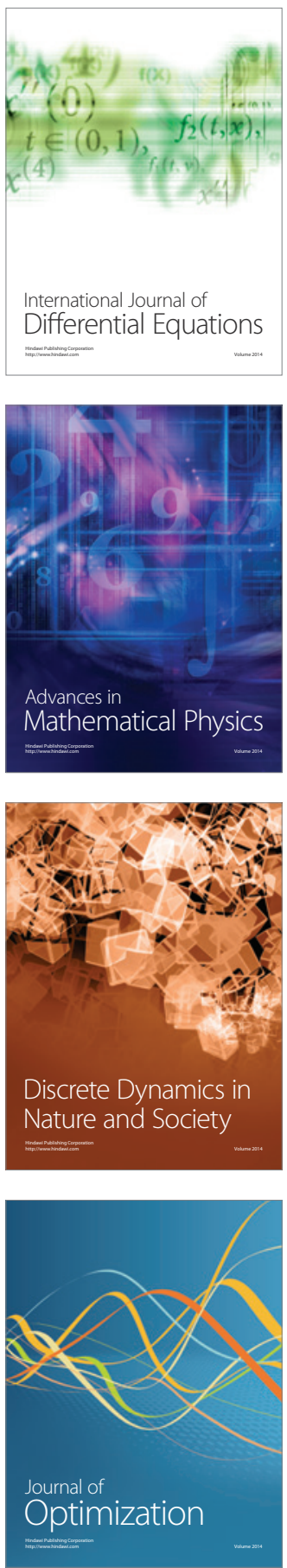